\title{
AN ADAPTIVE GENERALIZED MULTISCALE DISCONTINUOUS GALERKIN METHOD FOR HIGH-CONTRAST FLOW PROBLEMS*
}

\author{
ERIC T. $\mathrm{CHUNG}^{\dagger}$, YALCHIN EFENDIEV ${ }^{\ddagger}$, AND WING TAT LEUNG ${ }^{\S}$
}

\begin{abstract}
In this paper, we develop an adaptive generalized multiscale discontinuous Galerkin method (GMsDGM) for a class of high-contrast flow problems and derive a priori and a posteriori error estimates for the method. Based on the a posteriori error estimator, we develop an adaptive enrichment algorithm for our GMsDGM and prove its convergence. The adaptive enrichment algorithm gives an automatic way to enrich the approximation space in regions where the solution requires more basis functions, which are shown to perform well compared with a uniform enrichment. We also discuss an approach that adaptively selects multiscale basis functions by correlating the residual to multiscale basis functions (cf. [S. S. Chen, D. L. Donoho, and M. A. Saunders, SIAM Rev., 43 (2001), pp. 129-159]). The proposed error indicators are $L_{2}$-based and can be inexpensively computed, which makes our approach efficient. Numerical results are presented that demonstrate the robustness of the proposed error indicators.
\end{abstract}

Key words. adaptivity, high-contrast flow, multiscale finite element method, discontinuous Galerkin method, model reduction

AMS subject classifications. $65 \mathrm{~N} 12,65 \mathrm{~N} 30$

DOI. $10.1137 / 140986189$

1. Introduction. Model reduction techniques are often required for solving challenging multiscale problems that have multiple scales and high contrast. Many of these model reduction techniques perform the discretization of the problem on a coarse grid where coarse grid size is much larger than the fine grid discretization. The latter requires constructing reduced order models for the solution space on a coarse grid. Some of these techniques involve upscaled models (e.g., [14, 29]) or multiscale methods (e.g., $[2,5,15,19,20,21,22,6,11,7,9]$ ).

In this paper, we develop an adaptive generalized multiscale discontinuous Galerkin method (GMsDGM) for a class of high-contrast flow problems and derive a priori and a posteriori error estimates for the method. We propose an adaptive enrichment algorithm for our GMsDGM based on the a posteriori error estimator and prove its convergence. The enrichment is done using inexpensive $L_{2}$-based error indicators which allow adding more basis functions in an automatic way.

The generalized multiscale finite element method (GMsFEM), introduced in [16], is a generalization of the classical multiscale finite element method [23] by systematically enriching the coarse spaces and taking into account small scale information and complex input spaces. While GMsFEM uses continuous Galerkin methods as coarse grid solvers, the GMsDGM considered in this paper is based on the interior penalty

\footnotetext{
*Received by the editors September 10, 2014; accepted for publication (in revised form) June 8, 2018; published electronically August 7, 2018.

http://www.siam.org/journals/mms/16-3/98618.html

Funding: The first author was partially supported by the Hong Kong RGC General Research Fund (project 400813) and CUHK Direct Grant for Research 2013/14.

${ }^{\dagger}$ Department of Mathematics, Chinese University of Hong Kong, Hong Kong SAR (tschung@ math.cuhk.edu.hk).

${ }^{\ddagger}$ Department of Mathematics, Texas A\&M University, College Station, TX 77840; Numerical Porous Media SRI Center, King Abdullah University of Science and Technology (KAUST), Thuwal 23955-6900, Kingdom of Saudi Arabia (efendiev@math.tamu.edu).

$\S$ Department of Mathematics, Texas A\&M University, College Station, TX 77840 (leungwt@ math.tamu.edu).
} 
discontinuous Galerkin (IPDG) method as the coarse grid solver. The discontinuous Galerkin formulation has several key advantages in multiscale finite element methods (see [17]). The basis functions for the GMsDGM are totally decoupled across coarse element boundaries. In addition, the GMsDGM is constructed following the general framework on GMsFEM [16]. In particular, given a coarse grid partition of the computational domain, a snapshot space is defined for each coarse element. The snapshot space for a specific coarse element contains all functions defined in the underlying fine grid. A space reduction is then performed to obtain a much smaller space by means of spectral decomposition. Our analysis shows that we need two spectral problems for the space reduction. More precisely, the snapshot space is decomposed into two components, which consist of harmonic extensions and functions that are vanishing on coarse element boundaries. Two separate spectral problems are used to compute reduced spaces. An a priori error estimate is derived showing that the error is inverse proportional to the first eigenvalue corresponding to the first eigenfunction that is not used in the construction of the reduced space. We remark that similar results are obtained for GMsFEM [19, 18]. See also [8] for a review.

It is evident that different coarse elements need different numbers of basis functions in order to obtain accurate representation of the solution. For example, in less heterogeneous regions, one needs fewer basis functions compared to the regions with more heterogeneities and high contrast. It is therefore another objective of the paper to consider adaptive enrichment of basis functions. We derive an a posteriori error estimate for the GMsDGM. By using the error indicator, we construct an adaptive enrichment algorithm and prove its convergence. One important feature of our adaptive enrichment algorithm is the ability to adaptively select basis functions in the space of harmonic extensions and the space of functions vanishing on coarse element boundaries. In addition, the error indicators are $L_{2}$-based, which can be computed efficiently. This is an advantage over the $H^{-1}$-based adaptive enrichment algorithm developed for GMsFEM [10]. We also present a procedure for removing basis functions. Our analysis is based on the idea in $[3,25,10]$, and we do not consider the error due to the fine grid discretization of local problems and only study the errors due to the enrichment. In this regard, we assume that the error is largely due to coarse grid discretization. The fine grid discretization error can be considered in general (e.g., as in $[1,13]$ ) and this will give an additional error estimator. We remark that there are many related activities in designing a posteriori error estimates [12, 13, 1, 24, 26, 28] for global reduced models. The main difference is that our error estimators are based on a special local eigenvalue problem and use the eigenstructure of the offline space. We also discuss an approach that adaptively selects multiscale basis functions from the offline space by selecting a basis with the most correlation to the local residual (cf. [4]).

The rest of the paper is organized in the following way. In the next section, we present the basic idea of GMsDGM. The method is then detailed and analyzed in section 3. Then in section 4, we elaborate the adaptive algorithm and state the main convergence results related to this algorithm. In section 5, numerical results are illustrated to test the performance of this adaptive algorithm. The proofs of the main results are presented in section 6 .

2. Preliminaries. We will start this section with the problem settings and some notation. Let $D$ be the computational domain consisting of a medium modeled by the function $\kappa(x)$. The high-contrast flow problem concerned in this paper is

$$
-\operatorname{div}(\kappa(x) \nabla u)=f \quad \text { in } \quad D
$$


subject to the homogeneous Dirichlet boundary condition $u=g$ on $\partial D$. The main difficulty in numerically solving the above problem is that $\kappa(x)$ is highly heterogeneous with many scales and high contrast. We assume that $\kappa \geq 1$. In order to efficiently obtain an approximate solution to (1), we will need the notion of fine and coarse grids.

Consider a given triangulation $\mathcal{T}^{H}$ of the domain $D$ with mesh size $H>0$. We call $\mathcal{T}^{H}$ the coarse grid and $H$ the coarse mesh size. Elements of $\mathcal{T}^{H}$ are called coarse grid blocks and we use $N$ to denote the number of coarse grid blocks. The set of all coarse grid edges is denoted by $\mathcal{E}^{H}$. We also introduce a finer triangulation $\mathcal{T}^{h}$ of the computational domain $D$, obtained by a conforming refinement of the coarse grid $\mathcal{T}^{H}$. We call $\mathcal{T}^{h}$ the fine grid and $h>0$ the fine mesh size. We remark that the use of the conforming refinement is only to simplify the discussion of the methodology and is not a restriction of the method.

Now we present the framework of GMsDGM. The methodology consists of two main ingredients, namely, the construction of local basis functions and the global coarse grid level coupling. For the local basis functions, a snapshot space $V^{i \text {,snap }}$ is first constructed for each coarse grid block $K_{i} \in \mathcal{T}^{H}$. The snapshot space contains a large library of basis functions, which can be used to obtain a fine scale approximate solution to (1). A spectral problem is then solved in the snapshot space $V^{i \text {,snap }}$ and eigenfunctions corresponding to dominant modes are used as the final basis functions. The resulting space is called the local offline space $V^{i \text {,off }}$ for the $i$ th coarse grid block $K_{i}$. The global offline space $V^{\text {off }}$ is then defined as the linear span of all these $V^{i \text {,off }}$ for $i=1,2, \ldots, N$. This global offline space $V^{\text {off }}$ will be used as the approximation space of our discontinuous Galerkin method, which can be formulated as follows: find $u_{H}^{\mathrm{DG}} \in V^{\text {off }}$ such that

$$
a_{\mathrm{DG}}\left(u_{H}^{\mathrm{DG}}, v\right)=(f, v) \quad \forall v \in V^{\mathrm{off}},
$$

where the bilinear form $a_{\mathrm{DG}}$ is defined as

$$
a_{\mathrm{DG}}(u, v)=a_{H}(u, v)-\sum_{E \in \mathcal{E}^{H}} \int_{E}\left(\left\{\kappa \nabla u \cdot n_{E}\right\} \llbracket v \rrbracket+\left\{\kappa \nabla v \cdot n_{E}\right\} \llbracket u \rrbracket\right)+\sum_{E \in \mathcal{E}^{H}} \frac{\gamma}{h} \int_{E} \bar{\kappa} \llbracket u \rrbracket \llbracket v \rrbracket
$$

with

$$
a_{H}(u, v)=\sum_{K \in \mathcal{T}_{H}} a_{H}^{K}(u, v), \quad a_{H}^{K}(u, v)=\int_{K} \kappa \nabla u \cdot \nabla v,
$$

where $\gamma>0$ is a penalty parameter, and $n_{E}$ is a fixed unit normal vector defined on the coarse edge $E \in \mathcal{E}^{H}$. Note that, in (3), the average and the jump operators are defined in the classical way. Specifically, consider an interior coarse edge $E \in \mathcal{E}^{H}$ and let $K^{+}$and $K^{-}$be the two coarse grid blocks sharing the edge $E$. For a piecewise smooth function $G$, we define

$$
\{G\}=\frac{1}{2}\left(G^{+}+G^{-}\right), \quad \llbracket G \rrbracket=G^{+}-G^{-}, \quad \text { on } E,
$$

where $G^{+}=\left.G\right|_{K^{+}}$and $G^{-}=\left.G\right|_{K^{-}}$and we assume that the normal vector $n_{E}$ is pointing from $K^{+}$to $K^{-}$. Moreover, on the edge $E$, we define $\bar{\kappa}=\left(\kappa_{K^{+}}+\kappa_{K^{-}}\right) / 2$, where $\kappa_{K^{ \pm}}$is the maximum value of $\kappa$ over $K^{ \pm}$. For a coarse edge $E$ lying on the boundary $\partial D$, we define

$$
\{G\}=\llbracket G \rrbracket=G \quad \text { and } \quad \bar{\kappa}=\kappa_{K} \quad \text { on } E,
$$


where we always assume that $n_{E}$ is pointing outside of $D$. We note that the DG coupling (2) is the classical IPDG method [27] with our multiscale basis functions as the approximation space.

3. GMsDGM for high-contrast flow problems. In this section, we will give a detailed description of the method. We will first give the construction of the snapshot space and then give the definitions of the local spectral problems for the construction of the offline space. Furthermore, an a priori estimate of the method will be derived.

Let $K_{i} \in \mathcal{T}^{H}$ be a given coarse grid block. We will define two types of snapshot spaces. The first type of local snapshot space $V_{1}^{i \text {,snap }}$ for the coarse grid block $K_{i}$ is defined as the linear span of all harmonic extensions. Specifically, given a function $\delta_{k}$ defined on $\partial K_{i}$, we find $\psi_{k}^{i \text {,snap }} \in V_{h}\left(K_{i}\right)$ by

$$
\begin{aligned}
\int_{K_{i}} \kappa \nabla \psi_{k}^{i, \text { snap }} \cdot \nabla v & =0 \quad \forall v \in V_{h}^{0}\left(K_{i}\right), \\
\psi_{k}^{i, \text { snap }} & =\delta_{k} \quad \text { on } \partial K_{i},
\end{aligned}
$$

where $V_{h}\left(K_{i}\right)$ is the standard conforming piecewise polynomial finite element space with respect to the fine grid defined on $K_{i}, V_{h}^{0}\left(K_{i}\right)$ is the subspace of $V_{h}\left(K_{i}\right)$ containing functions vanishing on $\partial K_{i}$ and $\delta_{k}$ is piecewise polynomial on $\partial K_{i}$ with respect to the fine grid such that $\delta_{k}$ has the value one at the $k$ th fine grid degree of freedom (DOF) and value zero at all the remaining fine grid (DOF). The linear span of the above harmonic extensions is the local snapshot space $V_{1}^{i, \text { snap }}$, namely,

$$
V_{1}^{i, \text { snap }}=\operatorname{span}\left\{\psi_{k}^{i, \text { snap }}, \quad k=1,2, \ldots, M^{i, \text { snap }}\right\},
$$

where $M^{i, \text { snap }}$ is the number of basis functions in $V_{1}^{i \text {,snap }}$, which is also equal to the number of fine grid DOF on $\partial K_{i}$. The second type of local snapshot space $V_{2}^{i \text {,snap }}$ for the coarse grid block $K_{i}$ is defined as $V_{2}^{i \text {,snap }}=V_{h}^{0}\left(K_{i}\right)$. It is easy to see that $V_{h}\left(K_{i}\right)=V_{1}^{i \text {,snap }}+V_{2}^{i \text {,snap }}$, namely, the space $V_{h}\left(K_{i}\right)$ is decomposed as the sum of harmonic extensions and functions vanishing on the boundary $\partial K_{i}$. Moreover, the global snapshot space $V_{1}^{\text {snap }}$ is defined so that any $v \in V_{1}^{\text {snap }}$ if $\left.v\right|_{K_{i}} \in V_{1}^{i, \text { snap }}$. The space $V_{2}^{\text {snap }}$ is defined similarly.

We will perform dimension reductions on the above snapshot spaces by the use of some carefully selected spectral problems. Based on our analysis to be presented in this section, we define the spectral problem for $V_{1}^{i, \text { snap }}$ as finding eigenpairs $\left(\phi_{k}^{(i)}, \lambda_{1, k}^{(i)}\right)$, $k=1,2, \ldots, M^{i, \text { snap }}$, such that

$$
\int_{K_{i}} \kappa \nabla \phi_{k}^{(i)} \cdot \nabla v=\frac{\lambda_{1, k}^{(i)}}{H} \int_{\partial K_{i}} \widetilde{\kappa} \phi_{k}^{(i)} v \quad \forall v \in V_{1}^{i \text {,snap }},
$$

where $\widetilde{\kappa}$ is the maximum of $\bar{\kappa}$ over all coarse edges $E \in \partial K_{i}$. Moreover, we assume that

$$
\lambda_{1,1}^{(i)}<\lambda_{1,2}^{(i)}<\cdots<\lambda_{1, M^{i, \text { snap }}}^{(i)} .
$$

For the space $V_{2}^{i, \text { snap }}$, we define the spectral problem as finding eigenpairs $\left(\xi_{k}^{(i)}, \lambda_{2, k}^{(i)}\right)$, $k=1,2, \ldots$, such that

$$
\int_{K_{i}} \kappa \nabla \xi_{k}^{(i)} \cdot \nabla v=\frac{\lambda_{2, k}^{(i)}}{H^{2}} \int_{K_{i}} \kappa \xi_{k}^{(i)} v \quad \forall v \in V_{2}^{i, \text { snap }}
$$


where we also assume that

$$
\lambda_{2,1}^{(i)}<\lambda_{2,2}^{(i)}<\cdots .
$$

In the spectral problems (6) and (7), we will take respectively the first $l_{1, i}$ and $l_{2, i}$ eigenfunctions to form the offline space for the coarse grid block $K_{i}$. The local offline spaces are then defined as

$$
\begin{array}{ll}
V_{1}^{i, \text { off }}=\operatorname{span}\left\{\phi_{l}^{(i)},\right. & \left.l=1,2, \ldots, l_{1, i}\right\}, \\
V_{2}^{i, \text { off }}=\operatorname{span}\left\{\xi_{l}^{(i)}, \quad l=1,2, \ldots, l_{2, i}\right\} .
\end{array}
$$

We define $V^{i, \text { off }}=V_{1}^{i, \text { off }}+V_{2}^{i, \text { off }}$. The global offline space $V_{1}^{\text {off }}$ is defined so that the restriction of any function $v \in V_{1}^{\text {off }}$ on the coarse grid block $K_{i}$ belongs to $V_{1}^{i \text {,off }}$. The definition for $V_{2}^{\text {off }}$ is defined similarly. In addition, we define $V^{\text {off }}=V_{1}^{\text {off }}+V_{2}^{\text {off }}$. This space is used as the approximation space in (2). We remark that we assume the eigenvalues in the spectral problems (6) and (7) are simple only to simplify the notation. If there is a nonsimple eigenvalue, then multiple eigenfunctions correspond to this eigenvalue. In this case, we will include all these eigenfunctions in the space when this eigenvalue is chosen in our adaptive process.

Now we will analyze the method defined in (2). For any piecewise smooth function $u$, we define the DG-norm by

$$
\|u\|_{\mathrm{DG}}^{2}=a_{H}(u, u)+\sum_{E \in \mathcal{E}_{H}} \frac{\gamma}{h} \int_{E} \bar{\kappa} \llbracket u \rrbracket^{2} d s .
$$

Let $K$ be a coarse grid block and let $n_{\partial K}$ be the unit outward normal vector on $\partial K$. We denote $V_{h}(\partial K)$ by the restriction of the conforming space $V_{h}(K)$ on $\partial K$. For any $u \in V_{1}^{\text {snap }}$, the normal flux $\kappa \nabla u \cdot n_{\partial K}$ is understood as an element in $V_{h}(\partial K)$ and is defined by

$$
\int_{\partial K}\left(\kappa \nabla u \cdot n_{\partial K}\right) \cdot v=\int_{K} \kappa \nabla u \cdot \nabla \widehat{v}, \quad v \in V^{h}(\partial K),
$$

where $\widehat{v} \in V_{h}(K)$ is the harmonic extension of $v$ in $K$. By the Cauchy-Schwarz inequality,

$$
\int_{\partial K}\left(\kappa \nabla u \cdot n_{\partial K}\right) \cdot v \leq a_{H}^{K}(u, u)^{\frac{1}{2}} a_{H}^{K}(\widehat{v}, \widehat{v})^{\frac{1}{2}} .
$$

By an inverse inequality and the fact that $\widehat{v}$ is the harmonic extension of $v$

$$
a_{H}^{K}(\widehat{v}, \widehat{v}) \leq \kappa_{K} C_{\mathrm{inv}}^{2} h^{-1} \int_{\partial K}|v|^{2},
$$

where we recall that $\kappa_{K}$ is the maximum of $\kappa$ over $K$ and $C_{\mathrm{inv}}>0$ is the constant from inverse inequality. Thus,

$$
\int_{\partial K}\left(\kappa \nabla u \cdot n_{\partial K}\right) \cdot v \leq \kappa_{K}^{\frac{1}{2}} C_{\mathrm{inv}} h^{-\frac{1}{2}}\|v\|_{L^{2}(\partial K)} a_{H}^{K}(u, u)^{\frac{1}{2}} .
$$

This shows that

$$
\int_{\partial K}\left|\kappa \nabla u \cdot n_{\partial K}\right|^{2} \leq \kappa_{K} C_{\mathrm{inv}}^{2} h^{-1} a_{H}^{K}(u, u) .
$$

Copyright $@$ by SIAM. Unauthorized reproduction of this article is prohibited. 
We remark that the above steps show that the inequality (10) holds for any $u$ in the snapshot space $V_{1}^{\text {snap }}$. On the other hand, for any function $u \in V_{2}^{\text {snap }}$, one can show that (10) holds, with possibly a different constant $C_{\text {inv }}$, by using arguments based on inverse inequality. See also Lemma 2 in [9]. Furthermore, the relation (8) will be used in the proof of Theorem 3.2.

Our first step in the development of an a priori estimate is to establish the continuity and the coercivity of the bilinear form (3) with respect to the DG-norm.

Lemma 3.1. Assume that the penalty parameter $\gamma$ is chosen so that $\gamma>C_{\mathrm{inv}}^{2}$. The bilinear form $a_{D G}$ defined in (3) is continuous and coercive, that is,

$$
\begin{aligned}
& a_{D G}(u, v) \leq a_{1}\|u\|_{D G}\|v\|_{D G}, \\
& a_{D G}(u, u) \geq a_{0}\|u\|_{D G}^{2},
\end{aligned}
$$

for all $u, v$, where $a_{0}=1-C_{\mathrm{inv}} \gamma^{-\frac{1}{2}}>0$ and $a_{1}=1+C_{\mathrm{inv}} \gamma^{-\frac{1}{2}}$.

Proof. By the definition of $a_{\mathrm{DG}}$, we have

$$
a_{\mathrm{DG}}(u, v)=a_{H}(u, v)-\sum_{E \in \mathcal{E}^{H}} \int_{E}\left(\left\{\kappa \nabla u \cdot n_{E}\right\} \llbracket v \rrbracket+\left\{\kappa \nabla v \cdot n_{E}\right\} \llbracket u \rrbracket\right)+\sum_{E \in \mathcal{E}^{H}} \frac{\gamma}{h} \int_{E} \bar{\kappa} \llbracket u \rrbracket \llbracket v \rrbracket .
$$

Notice that

$$
a_{H}(u, v)+\sum_{E \in \mathcal{E}^{H}} \frac{\gamma}{h} \int_{E} \bar{\kappa} \llbracket u \rrbracket \cdot \llbracket v \rrbracket \leq\|u\|_{\mathrm{DG}}\|v\|_{\mathrm{DG}} .
$$

For an interior coarse edge $E \in \mathcal{E}^{H}$, we let $K^{+}, K^{-} \in \mathcal{T}^{H}$ be the two coarse grid blocks having the edge $E$. By the Cauchy-Schwarz inequality, we have

$$
\left|\int_{E}\left\{\kappa \nabla u \cdot n_{E}\right\} \cdot \llbracket v \rrbracket\right| \leq\left(h \int_{E}\left\{\kappa \nabla u \cdot n_{E}\right\}^{2}(\bar{\kappa})^{-1}\right)^{\frac{1}{2}}\left(\frac{1}{h} \int_{E} \bar{\kappa} \llbracket v \rrbracket^{2}\right)^{\frac{1}{2}} .
$$

Notice that

$h \int_{E}\left\{\kappa \nabla u \cdot n_{E}\right\}^{2}(\bar{\kappa})^{-1} \leq h\left(\int_{E}\left(\kappa^{+} \nabla u^{+} \cdot n_{E}\right)^{2}\left(\kappa_{K^{+}}\right)^{-1}+\int_{E}\left(\kappa^{-} \nabla u^{-} \cdot n_{E}\right)^{2}\left(\kappa_{K^{-}}\right)^{-1}\right)$,

where $u^{ \pm}=\left.u\right|_{K^{ \pm}}, \kappa^{ \pm}=\left.\kappa\right|_{K^{ \pm}}$. So, summing the above over all $E$ and by (10), we have

$$
h \sum_{E \in \mathcal{E}^{H}} \int_{E}\left\{\kappa \nabla u \cdot n_{E}\right\}^{2}(\bar{\kappa})^{-1} \leq h \sum_{K \in \mathcal{T}_{H}} \int_{\partial K}\left(\kappa \nabla u \cdot n_{\partial K}\right)^{2}\left(\kappa_{K}\right)^{-1} \leq C_{\mathrm{inv}}^{2} a_{H}(u, u) .
$$

Thus we have

$$
\sum_{E \in \mathcal{E}^{H}} \int_{E}\left\{\kappa \nabla u \cdot n_{E}\right\} \llbracket v \rrbracket \leq C_{\mathrm{inv}} a_{H}(u, u)^{\frac{1}{2}}\left(\sum_{E \in \mathcal{E}^{H}} \frac{1}{h} \int_{E} \bar{\kappa} \llbracket v \rrbracket^{2} d s\right)^{\frac{1}{2}} .
$$

Similarly, we have

$$
\sum_{E \in \mathcal{E}^{H}} \int_{E}\left\{\kappa \nabla v \cdot n_{E}\right\} \llbracket u \rrbracket \leq C_{\mathrm{inv}} a_{H}(v, v)^{\frac{1}{2}}\left(\sum_{E \in \mathcal{E}^{H}} \frac{1}{h} \int_{E} \bar{\kappa} \llbracket u \rrbracket^{2} d s\right)^{\frac{1}{2}} .
$$

Copyright (c) by SIAM. Unauthorized reproduction of this article is prohibited. 
Summing the above two inequalities, we have

$$
\sum_{E \in \mathcal{E}^{H}} \int_{E}\left(\left\{\kappa \nabla u \cdot n_{E}\right\} \llbracket v \rrbracket+\left\{\kappa \nabla v \cdot n_{E}\right\} \llbracket u \rrbracket\right) \leq C_{\mathrm{inv}} \gamma^{-\frac{1}{2}}\|u\|_{\mathrm{DG}}\|v\|_{\mathrm{DG}}
$$

This proves the continuity (11).

For the coercivity (12), we have

$$
a_{\mathrm{DG}}(u, u)=\|u\|_{\mathrm{DG}}^{2}-\sum_{E \in \mathcal{E}^{H}} \int_{E}\left(\left\{\kappa \nabla u \cdot n_{E}\right\} \cdot \llbracket u \rrbracket+\left\{\kappa \nabla u \cdot n_{E}\right\} \cdot \llbracket u \rrbracket\right) .
$$

By (15), we have

$$
a_{\mathrm{DG}}(u, u) \geq\left(1-C_{\mathrm{inv}} \gamma^{-\frac{1}{2}}\right)\|u\|_{\mathrm{DG}}^{2},
$$

which gives the desired result. let

In the following, we will prove an a priori estimate of the method (2). First, we

$$
V_{\mathrm{DG}}^{h}=\left\{v \in L^{2}(D):\left.v\right|_{K} \in V_{h}(K)\right\} .
$$

Let $u_{h} \in V_{\mathrm{DG}}^{h}$ be the fine grid solution which satisfies

$$
a_{\mathrm{DG}}\left(u_{h}, v\right)=(f, v) \quad \forall v \in V_{\mathrm{DG}}^{h} .
$$

It is well known that $u_{h}$ converges to the exact solution $u$ in the DG-norm as the fine mesh size $h \rightarrow 0$. Next, we define a projection $u_{1} \in V_{1}^{\text {snap }}$ of $u_{h}$ in the snapshot space by the following construction. For each coarse grid block $K_{i}$, the restriction of $u_{1}$ on $K_{i}$ is defined as the harmonic extension of $u_{h}$, that is,

$$
\begin{aligned}
\int_{K_{i}} \kappa \nabla u_{1} \cdot \nabla v & =0 & & \forall v \in V_{h}^{0}\left(K_{i}\right), \\
u_{1} & =u_{h} & & \text { on } \partial K_{i} .
\end{aligned}
$$

The following theorem gives an a priori estimate for the GMsDGM (2).

THEOREM 3.2. Let $u_{h} \in V_{D G}^{h}$ be the fine grid solution defined in (17) and $u_{H}$ be the GMsDGM solution defined in (2). Then we have

$$
\begin{aligned}
\left\|u_{h}-u_{H}\right\|_{D G}^{2} \leq C\left(\sum_{i=1}^{N}\right. & \frac{H}{\widetilde{\kappa} \lambda_{1, l_{1, i}+1}^{(i)}}\left(1+\frac{\gamma H}{h \lambda_{1, l_{1, i}+1}^{(i)}}\right) \int_{\partial K_{i}}\left(\kappa \nabla u_{1} \cdot n_{\partial K}\right)^{2} \\
& \left.+\sum_{K \in \mathcal{T}_{H}} \frac{H^{2}}{\lambda_{2, l_{2, i}+1}^{(i)}}\|f\|_{L^{2}(K)}^{2}+C_{\mathrm{inv}}^{2} \sum_{E \in \mathcal{E}^{H}} \frac{1}{h} \int_{E} \bar{\kappa} \llbracket u_{h} \rrbracket^{2}\right),
\end{aligned}
$$

where $u_{1}$ is defined in (18).

Proof. First, we write $u_{h}=u_{1}+u_{2}$, where $u_{2}=u_{h}-u_{1}$. Notice that, on each coarse grid block $K_{i}$, the functions $u_{1}$ and $u_{2}$ can be represented by

$$
u_{1}=\sum_{l=1}^{M_{i}} c_{l} \phi_{l}^{(i)} \quad \text { and } \quad u_{2}=\sum_{l \geq 1} d_{l} \xi_{l}^{(i)}
$$

Copyright (C) by SIAM. Unauthorized reproduction of this article is prohibited. 
where $M_{i}=M^{i \text {,snap }}$, and we assume that the functions $\phi_{l}^{(i)}$ and $\xi_{l}^{(i)}$ are normalized so that

$$
\int_{\partial K_{i}} \widetilde{\kappa}\left(\phi_{l}^{(i)}\right)^{2}=1 \text { and } \int_{K_{i}} \kappa\left(\xi_{l}^{(i)}\right)^{2}=1
$$

Notice that the functions $u_{1}$ and $u_{2}$ belong to the snapshot spaces $V_{1}^{\text {snap }}$ and $V_{2}^{\text {snap }}$, respectively. We will need two functions $\widehat{u}_{1}$ and $\widehat{u}_{2}$, which belong to the offline spaces $V_{1}^{\text {off }}$ and $V_{2}^{\text {off }}$, respectively. These functions are defined by

$$
\widehat{u}_{1}=\sum_{l=1}^{l_{1 . i}} c_{l} \phi_{l}^{(i)} \quad \text { and } \quad \widehat{u}_{2}=\sum_{l=1}^{l_{2 . i}} d_{l} \xi_{l}^{(i)} \quad \text { on } K_{i} .
$$

We remark that $\widehat{u}_{1}$ and $\widehat{u}_{2}$ are the truncation of $u_{1}$ and $u_{2}$ up to the eigenfunctions selected to form the offline space.

Next, we will find an estimate of $\left\|u_{1}-\widehat{u}_{1}\right\|_{\mathrm{DG}}$. Let $K_{i} \in \mathcal{T}^{H}$ be a given coarse grid block. Recall that the spectral problem to form $V_{1}^{i, \text { off }}$ is

$$
\int_{K_{i}} \kappa \nabla \phi_{k}^{(i)} \cdot \nabla v=\frac{\lambda_{1, k}^{(i)}}{H} \int_{\partial K_{i}} \widetilde{\kappa} \phi_{k}^{(i)} v \quad \forall v \in V_{1}^{i, \text { snap }}
$$

By the definition of the flux defined in (8), the above spectral problem can be represented as

$$
\int_{\partial K_{i}}\left(\kappa \nabla \phi_{k}^{(i)} \cdot n_{\partial K_{i}}\right) v d s=\frac{\lambda_{1, k}^{(i)}}{H} \int_{\partial K_{i}} \widetilde{\kappa} \phi_{k}^{(i)} v .
$$

By the definition of the DG-norm, the error $\left\|u_{1}-\widehat{u}_{1}\right\|_{\mathrm{DG}}$ can be estimated by

$$
\left\|\widehat{u}_{1}-u_{1}\right\|_{\mathrm{DG}}^{2} \leq \sum_{i=1}^{N}\left(\int_{K_{i}} \kappa\left|\nabla\left(\widehat{u}_{1}-u_{1}\right)\right|^{2}+\frac{\gamma}{h} \int_{\partial K_{i}} \widetilde{\kappa}\left(\widehat{u}_{1}-u_{1}\right)^{2}\right) .
$$

Note that, by (19), we have

$$
\int_{K_{i}} \kappa\left|\nabla\left(\widehat{u}_{1}-u_{1}\right)\right|^{2}=\sum_{l=l_{1, i}+1}^{M_{i}} \frac{\lambda_{1, l}^{(i)}}{H} c_{l}^{2} \leq \frac{H}{\lambda_{1, l_{1, i}+1}^{(i)}} \sum_{l=l_{1, i}+1}^{M_{i}}\left(\frac{\lambda_{1, l}^{(i)}}{H}\right)^{2} c_{l}^{2}
$$

and

$$
\left.\frac{1}{h} \int_{\partial K_{i}} \widetilde{\kappa}\left(\widehat{u}_{1}-u_{1}\right)\right)^{2}=\frac{1}{h} \sum_{l=l_{1, i}+1}^{M_{i}} c_{l}^{2} \leq \frac{H^{2}}{h\left(\lambda_{1, l_{1, i}+1}^{(i)}\right)^{2}} \sum_{l=l_{1, i}+1}^{M_{i}}\left(\frac{\lambda_{1, l}^{(i)}}{H}\right)^{2} c_{l}^{2} .
$$

Furthermore,

$$
\sum_{l=l_{1, i}+1}^{M_{i}}\left(\frac{\lambda_{1, l}^{(i)}}{H}\right)^{2} c_{l}^{2} \leq \sum_{l=1}^{M_{i}}\left(\frac{\lambda_{1, l}^{(i)}}{H}\right)^{2} c_{l}^{2}=(\widetilde{\kappa})^{-1} \int_{\partial K_{i}}\left(\kappa \nabla u_{1} \cdot n_{\partial K_{i}}\right)^{2} .
$$

Consequently, we obtain the following bound:

$$
\left\|u_{1}-\widehat{u}_{1}\right\|_{\mathrm{DG}}^{2} \leq \sum_{i=1}^{N} \frac{H}{\widetilde{\kappa} \lambda_{1, l_{1, i}+1}^{(i)}}\left(1+\frac{\gamma H}{h \lambda_{1, l_{1, i}+1}^{(i)}}\right) \int_{\partial K_{i}}\left(\kappa \nabla u_{1} \cdot n_{\partial K}\right)^{2} .
$$

Copyright $@$ by SIAM. Unauthorized reproduction of this article is prohibited. 
Next, we will find an estimate of $\left\|u_{2}-\widehat{u}_{2}\right\|_{\mathrm{DG}}$. By definition of the bilinear form $a_{D G}$,

$$
a_{D G}\left(u_{2}, v\right)=-a_{D G}\left(u_{1}, v\right)+(f, v)=\sum_{E \in \mathcal{E}^{H}} \int_{E}\left(\left\{\kappa \nabla v \cdot n_{E}\right\} \llbracket u_{1} \rrbracket\right)+(f, v),
$$

which holds for any $v \in V_{2}^{\text {snap }}$. In addition, by the fact that any function in $V_{2}^{\text {snap }}$ is zero on boundaries of coarse grid blocks, we have

$$
\left\|u_{2}-\widehat{u}_{2}\right\|_{\mathrm{DG}}^{2}=a_{D G}\left(u_{2}-\widehat{u}_{2}, u_{2}-\widehat{u}_{2}\right)=a_{D G}\left(u_{2}, u_{2}-\widehat{u}_{2}\right),
$$

where the last equality follows from the fact that the eigenfunctions of (7) are $\kappa$ orthogonal on every coarse grid block. Therefore we have

$$
\left\|u_{2}-\widehat{u}_{2}\right\|_{\mathrm{DG}}^{2}=\sum_{E \in \mathcal{E}^{H}} \int_{E}\left(\left\{\kappa \nabla\left(u_{2}-\widehat{u}_{2}\right) \cdot n_{E}\right\} \llbracket u_{1} \rrbracket\right)+\left(f, u_{2}-\widehat{u}_{2}\right) .
$$

The second term on the right-hand side of (21) can be estimated as

$$
\left(f, u_{2}-\widehat{u}_{2}\right) \leq \sum_{K \in \mathcal{T}^{H}}\|f\|_{L^{2}(K)}\left\|\kappa^{\frac{1}{2}}\left(u_{2}-\widehat{u}_{2}\right)\right\|_{L^{2}(K)} .
$$

By (7), for every $K_{i} \in \mathcal{T}^{H}$, we have

$$
\int_{K_{i}} \kappa\left|\left(u_{2}-\widehat{u}_{2}\right)\right|^{2}=\sum_{l \geq l_{2, i}+1} d_{l}^{2} \leq\left(\frac{H^{2}}{\lambda_{2, l_{2, i}+1}^{(i)}}\right) \sum_{l=l_{2, i}+1} \frac{\lambda_{2, l}^{(i)}}{H^{2}} d_{l}^{2}=\frac{H^{2}}{\lambda_{2, l_{2, i}+1}^{(i)}} \int_{K_{i}} \kappa\left|\nabla\left(u_{2}-\widehat{u}_{2}\right)\right|^{2} .
$$

For the first term on the right-hand side of (21), we use inequality (14) to conclude that

$$
\sum_{E \in \mathcal{E}^{H}} \int_{E}\left(\left\{\kappa \nabla\left(u_{2}-\widehat{u}_{2}\right) \cdot n_{E}\right\} \llbracket u_{1} \rrbracket\right) \leq C_{\mathrm{inv}} \gamma^{-\frac{1}{2}}\left\|u_{2}-\widehat{u}_{2}\right\|_{\mathrm{DG}}\left(\sum_{E \in \mathcal{E}^{H}} \frac{\gamma}{h} \int_{E} \bar{\kappa} \llbracket u_{1} \rrbracket^{2}\right)^{\frac{1}{2}} .
$$

Consequently, from $(21)$ and the fact that $\llbracket u_{1} \rrbracket=\llbracket u_{h} \rrbracket$ for all coarse edges, we obtain the following bound:

$$
\left\|u_{2}-\widehat{u}_{2}\right\|_{\mathrm{DG}}^{2} \leq C\left(C_{\mathrm{inv}}^{2} \sum_{E \in \mathcal{E}^{H}} \frac{1}{h} \int_{E} \bar{\kappa} \llbracket u_{h} \rrbracket^{2}+\sum_{K \in \mathcal{T}_{H}} \frac{H^{2}}{\lambda_{2, l_{2, i}+1}^{(i)}}\|f\|_{L^{2}(K)}^{2}\right) .
$$

Finally, we will prove the required error bound. By coercivity,

$$
\begin{aligned}
a_{0}\left\|\widehat{u}_{1}+\widehat{u}_{2}-u_{H}\right\|_{\mathrm{DG}}^{2} \leq & a_{\mathrm{DG}}\left(\widehat{u}_{1}+\widehat{u}_{2}-u_{H}, \widehat{u}_{1}+\widehat{u}_{2}-u_{H}\right) \\
= & a_{\mathrm{DG}}\left(\widehat{u}_{1}+\widehat{u}_{2}-u_{H}, \widehat{u}_{1}+\widehat{u}_{2}-u_{h}\right) \\
& +a_{\mathrm{DG}}\left(\widehat{u}_{1}+\widehat{u}_{2}-u_{H}, u_{h}-u_{H}\right) .
\end{aligned}
$$

Note that $a_{\mathrm{DG}}\left(\widehat{u}_{1}+\widehat{u}_{2}-u_{H}, u_{h}-u_{H}\right)=0$ since $\widehat{u}_{1}+\widehat{u}_{2}-u_{H} \in V^{\text {off }}$. Thus, by the triangle inequality, we have

$$
\left\|u_{h}-u_{H}\right\|_{\mathrm{DG}} \leq\left\|u_{h}-\widehat{u}\right\|_{\mathrm{DG}}+\left\|\widehat{u}-u_{H}\right\|_{\mathrm{DG}} \leq\left(1+a_{0}^{-\frac{1}{2}}\right)\left\|u_{h}-\widehat{u}\right\|_{\mathrm{DG}},
$$

where $\widehat{u}=\widehat{u}_{1}+\widehat{u}_{2}$. Hence, the desired bound is obtained by using (20) and (22).

Copyright $@$ by SIAM. Unauthorized reproduction of this article is prohibited. 
We remark that the term

$$
\sum_{i=1}^{N} \frac{H}{\widetilde{\kappa} \lambda_{1, l_{1, i}+1}^{(i)}}\left(1+\frac{\gamma H}{h \lambda_{1, l_{1, i}+1}^{(i)}}\right) \int_{\partial K_{i}}\left(\kappa \nabla u_{1} \cdot n_{\partial K}\right)^{2}
$$

corresponds to the error for the space $V_{1}^{\text {off }}$ and the term

$$
\sum_{K \in \mathcal{T}_{H}} \frac{H^{2}}{\lambda_{2, l_{2, i}+1}^{(i)}}\|f\|_{L^{2}(K)}^{2}
$$

corresponds to the error for the space $V_{2}^{\text {off }}$. Moreover, the term

$$
C_{\mathrm{inv}}^{2} \sum_{E \in \mathcal{E}^{H}} \frac{1}{h} \int_{E} \bar{\kappa} \llbracket u_{h} \rrbracket^{2}
$$

is the error in the fine grid solution $u_{h}$. This is the irreducible error, and an estimate of this can be derived following standard DG frameworks. In particular, the fine grid solution $u_{h}$ satisfies (17), which is the standard IPDG method with the use of the DG space defined in (16). One can find the error analysis of this method, for example, in [27]. More precisely, one can show that $\sum_{E \in \mathcal{E}^{H}} \frac{1}{h} \int_{E} \bar{\kappa} \llbracket u_{h} \rrbracket^{2} \leq C_{\text {err }} h^{2 p}$, where $p$ is the degree of the piecewise polynomial in $V_{h}(K)$. We note that the constant $C_{\text {err }}$ depends on the derivatives of the true solution and can be large. However, we assume that the fine mesh size $h$ is small enough to resolve all scales of the true solution so that this error term is small.

Remark 3.3. It is important to note that one can also replace (9) by

$$
a_{H}^{K}(\widehat{v}, \widehat{v}) \leq \Lambda_{K}^{\mathrm{snap}} \widetilde{\kappa} \int_{\partial K}|v|^{2},
$$

where $\Lambda_{K}^{\text {snap }}$ is the largest eigenvalue for the spectral problem (6). Therefore, (10) can be replaced by

$$
\int_{\partial K}\left|\kappa \nabla u \cdot n_{\partial K}\right|^{2} \leq \Lambda_{K}^{\mathrm{snap}} \widetilde{\kappa} a_{H}^{K}(u, u) .
$$

By following the above steps, we see that one can choose $\gamma$ in (23) so that

$$
\gamma>C_{\kappa} h \max _{K \subset \mathcal{T}^{H}} \Lambda_{K}^{\text {snap }}
$$

where the constant $C_{\kappa}$ is defined as

$$
C_{\kappa}=\max _{K \subset \mathcal{T}^{H}} \frac{\max _{E \subset \partial K} \bar{\kappa}}{\min _{E \subset \partial K} \bar{\kappa}} .
$$

We remark that this constant $C_{\kappa}$ is order one if we assume that every coarse element has a high contrast region.

One can take smaller values of $\gamma$ if oversampling is used (the oversampling method is discussed in section 5). The main idea of oversampling is to choose larger regions for computing snapshot vectors. For every coarse block $K_{i}$, we choose an enlarged region $K_{i}^{+}$and find oversampling snapshot functions $\psi_{k}^{i \text {,over }}$ by solving (37). We have 
TABLE 1

Left: oversampling basis. Right: no-oversampling basis.

\begin{tabular}{|c|c|c|c|}
\hline$h$ & $\Lambda_{K^{+}}^{\text {snap }}$ & $h$ & $\Lambda_{K}^{\text {snap }}$ \\
\hline $1 / 48$ & $1.0021 \mathrm{e}+03$ \\
\hline $1 / 96$ & $1.0193 \mathrm{e}+03$ \\
\hline $1 / 192$ & $1.3094 \mathrm{e}+03$ \\
\cline { 3 - 4 } & $1 / 48$ & $7.7650 \mathrm{e}+05$ \\
\hline $1 / 96$ & $1.6569 \mathrm{e}+06$ \\
\hline
\end{tabular}

performed numerical experiments and computed $\Lambda_{K}^{\text {snap }}$ with and without oversampling. Denote $\Lambda_{K^{+}}^{\text {snap }}$ to be the largest eigenvalue corresponding to the oversampled problem. In our numerical results (see Table 1), we have removed linearly dependent snapshot vectors with respect to the inner product corresponding to $\int_{\partial K}|v|^{2}$ before computing the largest eigenvalue. Our numerical results show that one can have about three orders of magnitude smaller value for $\Lambda_{K^{+}}^{\text {sna }}$ compared to $\Lambda_{K}^{\text {snap }}$. Moreover, our numerical results show a weak $h$-dependence for $\Lambda_{K^{+}}^{\text {snap }}$ as we decrease $h$, while $\Lambda_{K}^{\text {snap }}$ behaves as $h^{-1}$ (when no-oversampling is used).

Our error analysis holds when oversampling snapshot space is used. The term in (23) will become

$$
\sum_{i=1}^{N} \frac{H}{\widetilde{\kappa} \lambda_{1, l_{1, i}+1}^{(i)}}\left(1+\frac{\alpha C_{\kappa} \max _{K \subset \mathcal{T}^{H}} \Lambda_{K^{+}}^{\mathrm{snap}} H}{\lambda_{1, l_{1, i}+1}^{(i)}}\right) \int_{\partial K_{i}}\left(\kappa \nabla u_{1} \cdot n_{\partial K}\right)^{2}
$$

when $\gamma=\alpha C_{\kappa} h \max _{K \subset \mathcal{T}^{H}} \Lambda_{K^{+}}^{\text {snap }}$. If $\Lambda_{K^{+}}^{\text {snap }}$ is a weak function of $h$, e.g., if it is bounded with respect to $h$, then the terms involving $\Lambda_{K^{+}}^{\text {snap }}$ don't influence the error and the error is dominated by the first term. We emphasize that our discussions in this remark are based on our numerical studies and analytical study is difficult because it requires interior estimates for solutions. We plan to study this in the future.

Remark 3.4. From the remark above as well as (24) and (25), one sees that the penalty term in (3) can be replaced by

$$
\beta \Lambda_{\max }^{\operatorname{snap}} \int_{E} \bar{\kappa} \llbracket u \rrbracket \llbracket v \rrbracket,
$$

where $\beta$ is an $O(1)$ constant and $\Lambda_{\max }^{\text {snap }}:=\max _{K \subset \mathcal{T}^{H}} \Lambda_{K}^{\text {snap }}$. Moreover, it is easy to show that the result in Theorem 3.2 becomes

$$
\begin{aligned}
\left\|u_{h}-u_{H}\right\|_{\mathrm{DG}}^{2} \leq C\left(\sum_{i=1}^{N} \frac{1}{\widetilde{\kappa} \lambda_{1, l_{1, i}+1}^{(i)}}\left(1+\frac{\beta \Lambda_{\max }^{\mathrm{snap}}}{\lambda_{1, l_{1, i}+1}^{(i)}}\right) \int_{\partial K_{i}}\left(\kappa \nabla u_{1} \cdot n_{\partial K}\right)^{2}\right. \\
\left.+\sum_{K \in \mathcal{T}_{H}} \frac{H^{2}}{\lambda_{2, l_{2, i}+1}^{(i)}}\|f\|_{L^{2}(K)}^{2}+\sum_{E \in \mathcal{E}^{H}} \Lambda_{\max }^{\operatorname{snap}} \int_{E} \bar{\kappa} \llbracket u_{h} \rrbracket^{2}\right) .
\end{aligned}
$$

Remark 3.5. We note that while the solution can be well approximated by using our basis functions within coarse elements, the jumps of the solutions across coarse edges may not be well controlled (particularly for a small number of multiscale basis functions). One reason for this is because we use a constant penalty parameter $\gamma>$ 0 and a very large penalty factor (scaled as $h^{-1}$ ). In general, the solution has a multiscale structure on coarse edges, and the use of a multiscale penalty (instead of a constant penalty) is desirable and is important in order to capture the jumps of the solution (e.g., similar to hybridizable methods). Despite these limitations, the IPDG 
formulation used in this paper performs well in practical applications. The method is easy to implement in parallel using unstructured coarse meshes and avoids overlaps. Our paper presents an analysis of this method.

4. A posteriori error estimate and adaptive enrichment. In this section, we will derive an a posteriori error indicator for the error $u_{h}-u_{H}$ in energy norm. We will then use the error indicator to develop an adaptive enrichment algorithm. The a posteriori error indicator gives an estimate of the local error on the coarse grid blocks $K_{i}$, and we can then add basis functions to improve the solution. Our indicator consists of two components, which correspond to the errors made in the spaces $V_{1}^{\text {snap }}$ and $V_{2}^{\text {snap }}$. By using the indicator, one can determine adaptively which space has to be enriched. This section is devoted to the description of the a posteriori error indicator and the corresponding adaptive enrichment algorithm. The convergence analysis of the method will be given in section 6 .

Recall that $V_{\mathrm{DG}}^{h}$ is the fine scale DG finite element space, and the fine scale solution $u_{h}$ satisfies

$$
a_{\mathrm{DG}}\left(u_{h}, v\right)=(f, v) \quad \forall v \in V_{\mathrm{DG}}^{h} .
$$

Moreover, the GMsDGM solution $u_{H}$ satisfies

$$
a_{\mathrm{DG}}\left(u_{H}, v\right)=(f, v) \quad \forall v \in V^{\text {off }} .
$$

We remark that $V^{\text {off }} \subset V_{\mathrm{DG}}^{h}$. Next we will give the definitions of the residuals.

Definitions of residuals. Let $K_{i}$ be a given coarse grid block. We will define two residuals corresponding to the two types of snapshot spaces. First, on the space $V_{1}^{i, \text { snap }}$, we define the following linear functional:

$$
R_{1, i}(v)=\int_{K_{i}} f v-a_{\mathrm{DG}}\left(u_{H}, v\right), \quad v \in V_{1}^{i, \text { snap }} .
$$

Similarly, on the space $V_{2}^{i \text {,snap }}$, we define the following linear functional:

$$
R_{2, i}(v)=\int_{K_{i}} f v-a_{\mathrm{DG}}\left(u_{H}, v\right), \quad v \in V_{2}^{i, \text { snap }} .
$$

These residuals measure how well the solution $u_{H}$ satisfies the fine scale equation (27). Furthermore, on the snapshot spaces $V_{1}^{i \text {,snap }}$ and $V_{2}^{i, \text { snap }}$, we define the following norms:

$$
\|v\|_{V_{1}\left(K_{i}\right)}^{2}=H^{-1} \int_{\partial K_{i}} \widetilde{\kappa} v^{2} \text { and }\|v\|_{V_{2}\left(K_{i}\right)}^{2}=H^{-2} \int_{K_{i}} \kappa v^{2},
$$

respectively. The norms of the linear functionals $R_{1, i}$ and $R_{2, i}$ are defined in the standard way, namely,

$$
\left\|R_{1, i}\right\|=\sup _{v \in V_{1}^{i, \text { snap }}} \frac{\left|R_{1, i}(v)\right|}{\|v\|_{V_{1}\left(K_{i}\right)}} \quad \text { and } \quad\left\|R_{2, i}\right\|=\sup _{v \in V_{2}^{i, \text { snap }}} \frac{\left|R_{2, i}(v)\right|}{\|v\|_{V_{2}\left(K_{i}\right)}} .
$$

The norms $\left\|R_{1, i}\right\|$ and $\left\|R_{2, i}\right\|$ give estimates on the sizes of fine scale residual errors with respect to the spaces $V_{1}^{i, \text { snap }}$ and $V_{2}^{i, \text { snap }}$.

We recall that, for each coarse grid block $K_{i}$, the eigenfunctions of the spectral problem (6) corresponding to the eigenvalues $\lambda_{1,1}^{(i)}, \ldots, \lambda_{1, l_{1, i}}^{(i)}$ and the eigenfunctions 
of the spectral problem (7) corresponding to the eigenvalues $\lambda_{2,1}^{(i)}, \ldots, \lambda_{2, l_{2, i}}^{(i)}$ are used in the construction of $V^{\text {off }}$. In addition, the energy error in this section and section 6 is measured by $\|u\|_{a}^{2}=a_{\mathrm{DG}}(u, u)$, which is equivalent to the DG-norm.

In section 6 , we will prove the following theorem, and we see that the norms $\left\|R_{j, i}\right\|$ give indications on the size of the energy norm error $\left\|u_{h}-u_{H}\right\|_{a}$.

TheOrem 4.1. Let $u_{h}$ and $u_{H}$ be the solutions of (27) and (28), respectively. Then

$$
\left\|u_{h}-u_{H}\right\|_{a}^{2} \leq C_{e r r} \sum_{i=1}^{N} \sum_{j=1}^{2}\left\|R_{j, i}\right\|^{2}\left(\lambda_{j, l_{j, i}+1}^{(i)}\right)^{-1} .
$$

where $C_{\text {err }}$ is a uniform constant.

We will now present the adaptive enrichment algorithm. We use $m \geq 1$ to represent the enrichment level, $V^{\text {off }}(m)$ to represent the solution space at level $m$, and $u_{H}^{m}$ to represent the GMsDGM solution at the enrichment level $m$. For each coarse grid block $K_{i}$, we use $l_{j, i}^{m}$ to represent the number of eigenfunctions in $V_{j}^{i \text { off }}$ used at the enrichment level $m$ for the coarse region $K_{i}$. Assume that the initial offline space $V^{\text {off }}(0)$ is given.

Adaptive enrichment algorithm. Choose $0<\theta<1$. For each $m=0,1, \ldots$,

1. Step 1: Find the solution in the current space. That is, find $u_{H}^{m} \in V^{\text {off }}(m)$ such that

$$
a_{\mathrm{DG}}\left(u_{H}^{m}, v\right)=(f, v) \quad \forall v \in V^{\mathrm{off}}(m) .
$$

2. Step 2: Compute the local residuals. For each coarse grid block $K_{i}$, we compute

$$
\eta_{j, i}^{2}=\left\|R_{j, i}\right\|^{2}\left(\lambda_{j, l_{j, i}^{m}+1}^{(i)}\right)^{-1}, \quad j=1,2
$$

where the residuals and their norms are defined in (29)-(32). In particular, the residuals are the $L^{2}$ projections with respect to the inner products (31) and their norms are $L^{2}$ norms of these projections. After we computed the residual norms, we reenumerated the above $2 N$ residuals in decreasing order, that is, $\eta_{1}^{2} \geq \eta_{2}^{2} \geq \cdots \geq \eta_{2 N}^{2}$, where we adopted single index notation. That is, each single index $k$ corresponds to a double index $(j, i)$ for the $i$ th coarse block $K_{i}$ and the space $V_{j}^{i, \text { off }}$.

3. Step 3: Find the coarse grid blocks and spaces where enrichment is needed. We choose the smallest integer $k$ such that

$$
\theta \sum_{J=1}^{2 N} \eta_{J}^{2} \leq \sum_{J=1}^{k} \eta_{J}^{2}
$$

The above is the standard Dorfler marking strategy. It is also a criterion to select the coarse regions with large errors. The numbers of regions and spaces to enrich are determined by the parameter $\theta$.

4. Step 4: Enrich the space. For each $J=1,2, \ldots, k$, chosen by the above criterion, we add basis functions in $V_{j}^{i, \text { off }}$ according to the following rule. Let $s$ be the smallest positive integer such that $\lambda_{j, l_{j, i}^{m}+s+1}^{(i)}$ is large enough (see the proof of Theorem 4.2) compared with $\lambda_{j, l_{j, i}^{m}+1}^{(i)}$. Then we will add 
the eigenfunctions $\phi_{k}^{(i)}$ or $\xi_{k}^{(i)}$ from the spectral problem (6) or (7) for $k=$ $l_{j, i}^{m}+1, \ldots, l_{j, i}^{m}+s$, depending on the value of $j$, in the construction of the basis functions. We note that $j=1$ or 2 corresponds to the space defined by eigenfunctions the spectral problem (6) or (7). The resulting space is denoted as $V^{\text {off }}(m+1)$.

We remark that the choice of $s$ above will ensure the convergence of the enrichment algorithm, and in practice, the value of $s$ is easy to obtain. Moreover, contrary to classical adaptive refinement methods, the total number of basis functions that we can add is bounded by the dimension of the snapshot space. Thus, the condition (35) can be modified as follows. We choose the smallest integer $k$ such that

$$
\theta \sum_{J=1}^{2 N} \eta_{J}^{2} \leq \sum_{J \in I} \eta_{J}^{2}
$$

where the index set $I$ is a subset of $\{1,2, \ldots, k\}$.

Finally, we state the convergence theorem.

THEOREM 4.2. There are sequence $\left\{L_{m}\right\}_{m=1}^{M}$ and positive constants $\tau, \epsilon_{0}, \rho$ independent of $m$ such that the following contracting property holds:

$$
\left\|u_{h}-u_{H}^{m+1}\right\|_{a}^{2}+\frac{\tau}{1+\tau L_{m+1}} \sum_{J=1}^{2 N}\left(S_{J}^{m+1}\right)^{2} \leq \varepsilon\left(\left\|u_{h}-u_{H}^{m}\right\|_{a}^{2}+\frac{\tau}{1+\tau L_{m}} \sum_{J=1}^{2 N}\left(S_{J}^{m}\right)^{2}\right) .
$$

Note that $0<\varepsilon<1$ and

$$
\varepsilon=1-\epsilon_{0} \theta^{2} \frac{\tau}{2 C_{\mathrm{err}}\left(1+\tau L_{M}\right)},
$$

where we can take any value of $\theta$ with the following condition:

$$
0<\theta<\min \left\{1,\left(\frac{C_{\mathrm{err}}\left(1+\tau L_{M}\right)}{\tau}\right)^{\frac{1}{2}}\right\} .
$$

We remark that the precise definitions of $S_{J}^{m}$ as well as the constants $\tau, \epsilon_{0}, \rho$ and the sequence $L_{m}$ are given in section 6 .

5. Numerical results. In this section, we will present some numerical examples to demonstrate the performance of the adaptive enrichment algorithm. The domain $\Omega$ is taken as the unit square $[0,1]^{2}$ and is divided into $16 \times 16$ coarse blocks consisting of uniform squares. Each coarse block is then divided into $32 \times 32$ fine blocks consisting of uniform squares. Consequently, the whole domain is partitioned by $512 \times 512$ fine grid blocks. We will use the following error quantities to compare the accuracy of our algorithm:

$$
\begin{gathered}
e_{2}=\frac{\left\|u_{H}-u_{h}\right\|_{L^{2}(\Omega)}}{\left\|u_{h}\right\|_{L^{2}(\Omega)}}, e_{a}=\frac{\sqrt{a_{\mathrm{DG}}\left(u_{H}-u_{h}, u_{H}-u_{h}\right)}}{\sqrt{a_{\mathrm{DG}}\left(u_{h}, u_{h}\right)}}, \\
e_{2}^{\text {snap }}=\frac{\left\|u_{H}-u_{\text {snap }}\right\|_{L^{2}(\Omega)}}{\left\|u_{\text {snap }}\right\|_{L^{2}(\Omega)}}, e_{a}^{\text {snap }}=\frac{\sqrt{a_{\mathrm{DG}}\left(u_{H}-u_{\text {snap }}, u_{H}-u_{\text {snap }}\right)}}{\sqrt{a_{\mathrm{DG}}\left(u_{\text {snap }}, u_{\text {snap }}\right)}},
\end{gathered}
$$

where $u_{H}$ and $u_{h}$ are the GMsDGM and the fine grid solutions, respectively. Moreover, $u_{\text {snap }}$ is the snapshot solution obtained by using all snapshot functions generated by an oversampling strategy; see below. 
We consider the permeability field $\kappa$ which is shown in Figure 1. The boundary condition is set to be bilinear, $g=x_{1} x_{2}$. We will consider two examples with two different source functions $f$. We will compare the result of $V_{1}$ enrichment, $V_{1}-$ $V_{2}$ enrichment, oversampling basis enrichment, uniform enrichment, and the exact indicator enrichment. The following gives the details of these enrichments.

- $V_{1}$ enrichment: We use the error indicator $\eta_{1, i}^{2}$ to perform the adaptive algorithm by enriching the basis functions in $V_{1}$ space only, that is, basis functions obtained by the first spectral problem (6). We use four basis functions from (6) and zero basis function from (7) in the initial step.

- $V_{1}-V_{2}$ enrichment: We use both the error indicators $\eta_{1, i}^{2}, \eta_{2, i}^{2}$ to perform the adaptive algorithm by enriching the basis functions in both $V_{1}$ and $V_{2}$ spaces, that is, basis functions from both spectral problems (6) and (7). We use four basis functions from (6) and zero basis function from (7) in the initial step.

- Oversampling enrichment: For every coarse block $K_{i}$, we choose an enlarged region $K_{i}^{+}$(in the examples presented below, we enlarge the coarse block in each direction by a length $H$, that is, $K_{i}^{+}$is a $3 \times 3$ coarse block with $K_{i}$ at the center). Then we find oversampling snapshot functions $\psi_{k}^{i, \text { over }} \in V_{h}\left(K_{i}^{+}\right)$ by solving

$$
\begin{aligned}
\int_{K_{i}} \kappa \nabla \psi_{k}^{i, \text { over }} \cdot \nabla v & =0 \quad \forall v \in V_{h}^{0}\left(K_{i}^{+}\right), \\
\psi_{k}^{i, \text { over }} & =\delta_{k} \quad \text { on } \partial K_{i}^{+} .
\end{aligned}
$$

The linear span of these snapshot functions is called $V^{i \text {,over }}$. Then we choose 40 dominant oversampling basis functions by the proper orthogonal decomposition method. Specifically, we solve the eigenvalue problem

$$
\int_{K_{i}} \psi_{k}^{i, \text { over }} v=\lambda_{k}^{i} \int_{\partial K_{i}^{+}} \psi_{k}^{i, \text { over }} v \quad \forall v \in V^{i, \text { over }}
$$

and choose the first 40 eigenfunctions with largest eigenvalues. Then we use these 40 functions as boundary conditions in (5) and repeat the remaining construction of the offline space.

- Uniform enrichment: We enrich the basis functions in $V_{1}$ space uniformly with four basis functions from the $V_{1}$ space in the initial step.

- The exact indicator enrichment: We use the exact error as the error indicator to perform the adaptive algorithm by enriching the basis functions in $V_{1}$ space only with four basis functions in the space $V_{1}$ in the initial step. Here, the exact error is defined as $\left\|u-u_{H}\right\|_{a}$.

Example 1. In our first example, we take the source function $f=1$. The fine grid solution is shown in Figure 1. In Tables 2 and 3, we present the convergence history of our algorithm for enriching in $V_{1}$ space only, enriching in both $V_{1}$ and $V_{2}$ spaces, and enriching by the oversampling basis functions. We remark that, in the presentation of our results, DOF means the total number of basis functions used in the whole domain. We see from Table 2 that the behavior of enriching in $V_{1}$ space only and enriching in both $V_{1}$ and $V_{2}$ spaces is similar. The is due to the fact that the source function $f$ is a constant function, and the space $V_{2}$ will not help to improve the solution. This is consistent with classical theory that basis functions obtained by harmonic extensions are good enough to approximate the solution. In Table 3, the convergence behavior is shown for the oversampling case, and we see again that a 

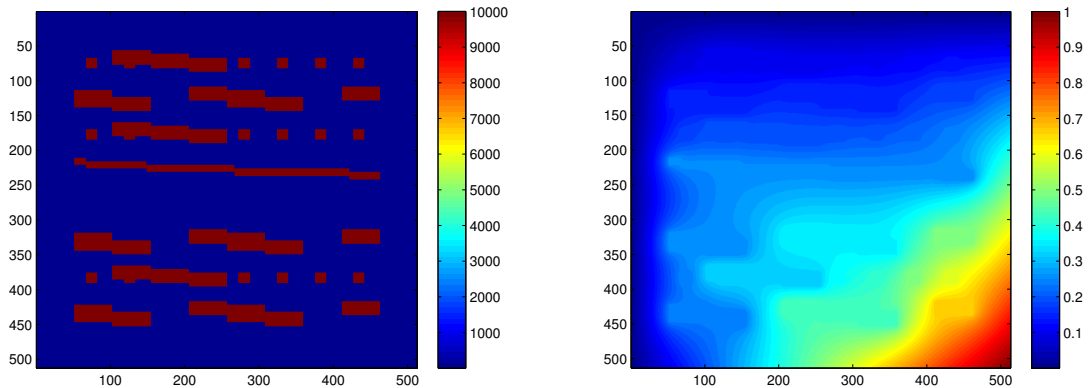

FIG. 1. Left: Permeability field $\kappa$. Right: Fine grid solution with $f=1$.

TABLE 2

Convergence history with $\theta=0.4$. Left: Enrich in $V_{1}$ space only. Right: Enrich in both $V_{1}$ and $V_{2}$ spaces.

\begin{tabular}{|c|c|c|}
\hline DOF & $e_{2}$ & $e_{a}$ \\
\hline 1024 & 0.1082 & 0.0479 \\
\hline 1769 & 0.0456 & 0.0178 \\
\hline 2403 & 0.0156 & 0.0105 \\
\hline 3135 & 0.0070 & 0.0067 \\
\hline 5607 & 0.0016 & 0.0031 \\
\hline
\end{tabular}

\begin{tabular}{|c|c|c|}
\hline DOF & $e_{2}$ & $e_{a}$ \\
\hline 1024 & 0.1082 & 0.0479 \\
\hline 1639 & 0.0802 & 0.0239 \\
\hline 2584 & 0.0194 & 0.0114 \\
\hline 3822 & 0.0061 & 0.0063 \\
\hline 5660 & 0.0021 & 0.0037 \\
\hline
\end{tabular}

TABLE 3

Left: Convergence history for oversampling basis with $\theta=0.4$ and enrichment in $V_{1}$ space only. Right: Convergence history for uniform enrichment in $V_{1}$ space only.

\begin{tabular}{|c|c|c|c|c|}
\hline $\mathrm{DOF}$ & $e_{2}$ & $e_{a}$ & $e_{2}^{\text {snap }}$ & $e_{a}^{\text {snap }}$ \\
\hline 1024 & 0.0940 & 0.0469 & 0.0939 & 0.0469 \\
\hline 1975 & 0.0204 & 0.0121 & 0.0202 & 0.0121 \\
\hline 2648 & 0.0087 & 0.0077 & 0.0084 & 0.0077 \\
\hline 3422 & 0.0046 & 0.0056 & 0.0043 & 0.0056 \\
\hline 6748 & 0.0009 & 0.0022 & 0.0006 & 0.0020 \\
\hline
\end{tabular}

\begin{tabular}{|c|c|c|}
\hline DOF & $e_{2}$ & $e_{a}$ \\
\hline 1024 & 0.1082 & 0.0479 \\
\hline 2048 & 0.0671 & 0.0199 \\
\hline 3328 & 0.0423 & 0.0150 \\
\hline 5888 & 0.0161 & 0.0059 \\
\hline 8448 & 0.0128 & 0.0044 \\
\hline
\end{tabular}

clear convergence is obtained. For this case, we use 40 snapshot basis functions per coarse grid block, giving a total DOF of 10240, and the corresponding snapshot errors (that is, the difference between the solution obtained by these 10240 basis functions and the solution $u_{h}$ ) of $4.5195 \times 10^{-4}$ and $9.8935 \times 10^{-4}$ in relative $L^{2}$ norm and relative $a$-norm, respectively. In addition, we observe that the oversampling basis provides more efficient representation of the solution than the nonoversampling basis. To further demonstrate the efficiency of our algorithm, we compare our result with the uniform enrichment scheme. The convergence history for using uniform enrichment is shown in Table 3, and we see that our adaptive enrichment algorithm performs much better than uniform enrichment. Finally, a comparison among all the above cases and the enrichment by exact error is shown in Figure 2, in which the energy error is plotted against DOF. From the figure, we clearly see that our enrichment algorithm performs much better than uniform enrichment. Moreover, our enrichment algorithm performs equally well compared with enrichment by the exact error. This shows that our indicator is both reliable and efficient.

Next, we will numerically demonstrate in Figure 3 the result of Theorem 3.2. In particular, we will show that the error decay is inversely proportional to the smallest 


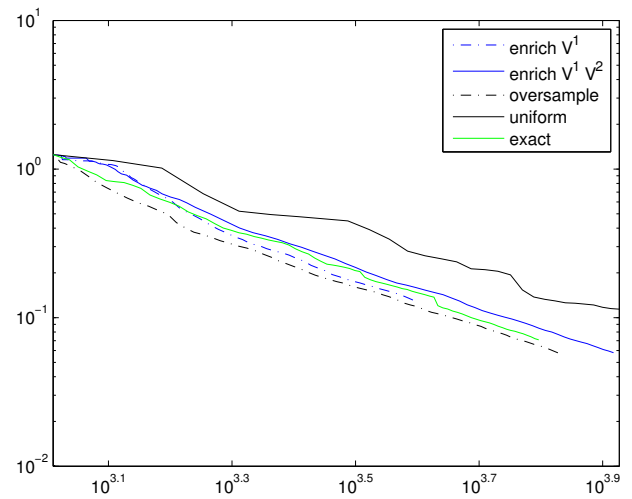

FIG. 2. A comparison of different ways of enrichment.
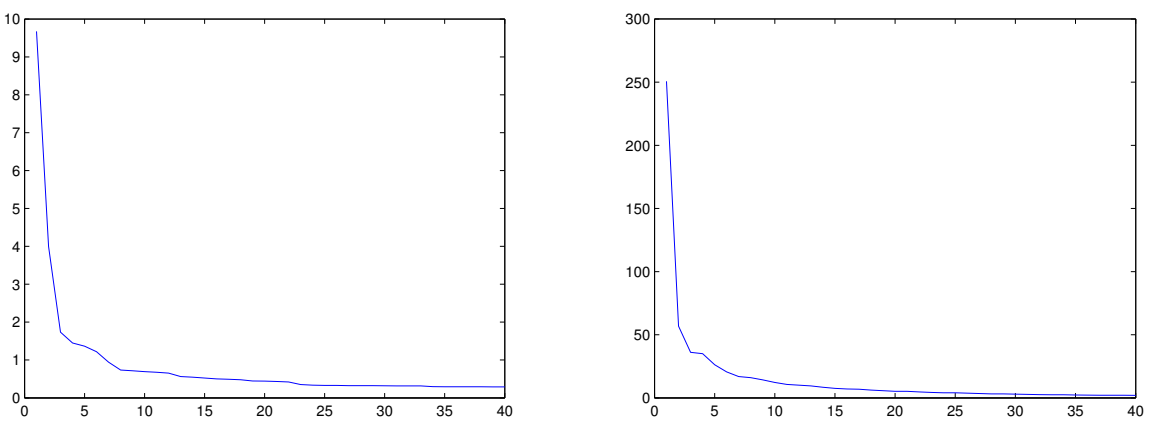

FIG. 3. Relation of error and eigenvalue decays. The correlation coefficient of these two curves is 0.9863. Left: Relative error decay. Right: Eigenvalue decay.

eigenvalue corresponding to the unused eigenfunctions. To better show this relation, we will consider basis function enrichment only in the $V_{1}$ space and we fix the number of basis functions in the $V_{2}$ space. Thus, we consider only the relation between error decay and the reciprocal of eigenvalues from the $V_{1}$ space. In the left graph of Figure 3 , the decay in relative error with respect to the number of basis functions per coarse element is shown, and in the right graph of Figure 3, the decay reciprocal of the eigenvalue with respect to the number of basis functions per coarse element is shown. We can see that the decay behaviors are very similar. Indeed, the correlation of these two curves is 0.9863 , which confirms our result in Theorem 3.2. We remark that we conducted a similar test for the space $V_{2}$ and obtained the same conclusion.

Finally, we will show numerically that the error indicator (29)-(30) gives a good approximation of the error. In Figure 4, we plot the ratio of error to residual with respect to the dimension of the approximation space, which contains both basis functions from $V_{1}$ and $V_{2}$. The error and the residual are the quantities given in (33). We see that the ratio remains bounded and $O(1)$ and is independent of the dimension of the approximation space. This shows that the constant in (33) is $O(1)$. This also shows that the residual gives a good indication of the level of error. 


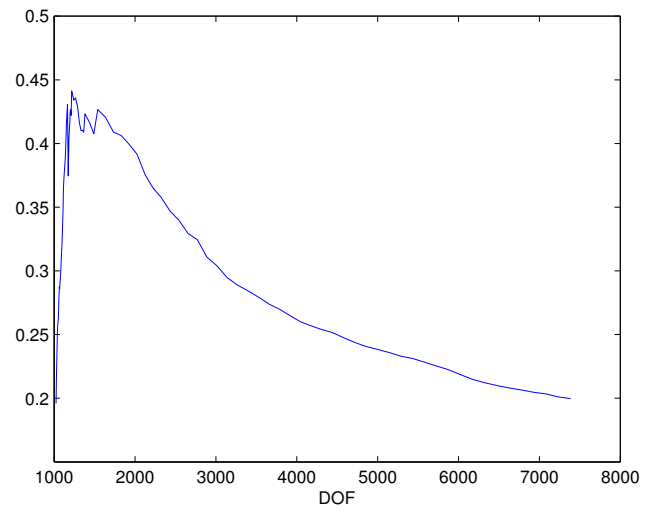

FIG. 4. The ratio of error to residual with respect to the dimension of the approximation space.
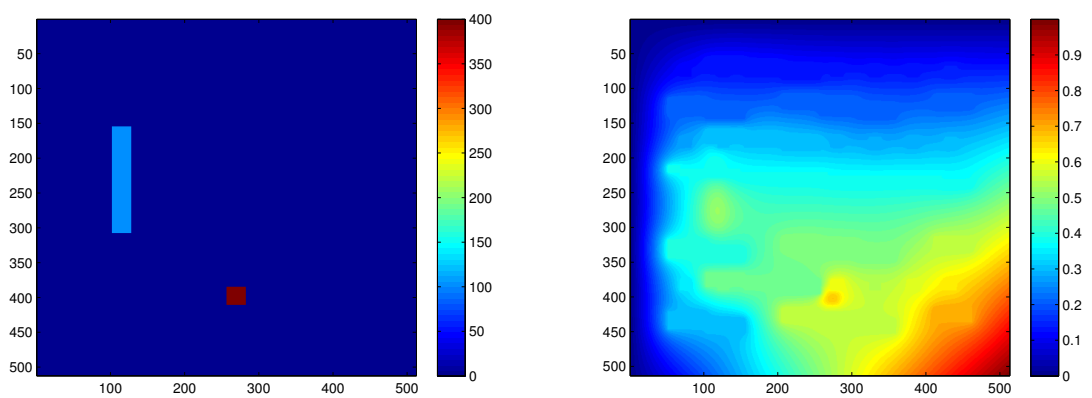

FIG. 5. Left: The source function $f$ for the second example. Right: The fine grid solution.

Example 2. In our second example, we will take the source $f$ to be the function shown in the left plot of Figure 5 and the corresponding fine grid solution shown in the right plot of Figure 5. In Tables 4 and 5 , we present the convergence history of our algorithm for enriching in $V_{1}$ space only, enriching in both $V_{1}$ and $V_{2}$ spaces, and enriching by oversampling basis. We see from Table 4 that enrichment in both $V_{1}$ and $V_{2}$ spaces provides much more efficient methods than enrichment in $V_{1}$ space only. In particular, for an error level of approximately $1 \%$, we see that enrichment in both $V_{1}$ and $V_{2}$ spaces requires $3144 \mathrm{DOF}$, while enrichment in $V_{1}$ space only requires 3483 DOF. In Table 5 , the convergence behavior is shown for the oversampling case, and we see again that a clear convergence is obtained. For this case, we use 40 snapshot basis functions per coarse grid block, giving a total DOF of 10240, and the corresponding snapshot errors (that is, the difference between the solution obtained by these 10240 basis functions and the solution $u_{h}$ ) of 0.0078 and 0.0093 in relative $L^{2}$ norm and relative $a$-norm, respectively. In addition, we observe again that the oversampling basis provides more efficient representation of the solution than the nonoversampling basis. To further demonstrate the efficiency of our algorithm, we compare our results with the uniform enrichment scheme. The result for using uniform enrichment is shown in Table 5 and we clearly observe that our adaptive method is more efficient. Moreover, a comparison of the performance of various strategies is shown in Figure 6, where the errors against DOF are plotted. From the figure, we see that our method 
TABLE 4

Convergence history with $\theta=0.4$. Left: Enrich in $V_{1}$ space only. Right: Enrich in both $V_{1}$ and $V_{2}$ spaces.

\begin{tabular}{|c|c|c|}
\hline DOF & $e_{2}$ & $e_{a}$ \\
\hline 1024 & 0.2052 & 0.0554 \\
\hline 2028 & 0.0362 & 0.0191 \\
\hline 2717 & 0.0152 & 0.0140 \\
\hline 3483 & 0.0111 & 0.0118 \\
\hline 5116 & 0.0084 & 0.0102 \\
\hline
\end{tabular}

\begin{tabular}{|c|c|c|}
\hline DOF & $e_{2}$ & $e_{a}$ \\
\hline 1024 & 0.2052 & 0.0554 \\
\hline 2023 & 0.0486 & 0.0206 \\
\hline 3144 & 0.0113 & 0.0105 \\
\hline 4456 & 0.0050 & 0.0066 \\
\hline 7407 & 0.0013 & 0.0034 \\
\hline
\end{tabular}

TABLE 5

Left: Convergence history for oversampling basis with $\theta=0.4$ and enrichment in $V_{1}$ space only. Right: Convergence history for uniform enrichment in $V_{1}$ space only.

\begin{tabular}{|c|c|c|c|c|}
\hline DOF & $e_{2}$ & $e_{a}$ & $e_{2}^{\text {snap }}$ & $e_{a}^{\text {snap }}$ \\
\hline 1024 & 0.1882 & 0.0540 & 0.1865 & 0.0532 \\
\hline 1926 & 0.0296 & 0.0182 & 0.0269 & 0.0156 \\
\hline 2626 & 0.0137 & 0.0135 & 0.0098 & 0.0098 \\
\hline 3368 & 0.0105 & 0.0116 & 0.0057 & 0.0070 \\
\hline 6677 & 0.0080 & 0.0097 & 0.0007 & 0.0025 \\
\hline
\end{tabular}

\begin{tabular}{|c|c|c|}
\hline DOF & $e_{2}$ & $e_{a}$ \\
\hline 1024 & 0.2052 & 0.0554 \\
\hline 2048 & 0.0923 & 0.0282 \\
\hline 3328 & 0.0659 & 0.0215 \\
\hline 5888 & 0.0278 & 0.0135 \\
\hline 8448 & 0.0226 & 0.0121 \\
\hline
\end{tabular}

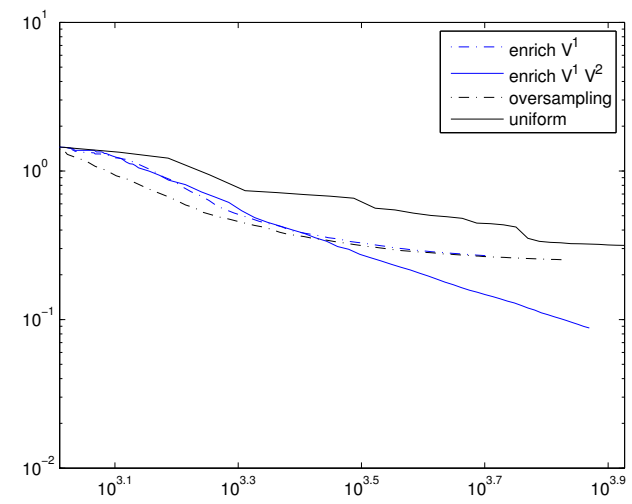

FIG. 6. A comparison of different ways of enrichment.

is much better than uniform enrichment. Furthermore, enrichment in both $V_{1}$ and $V_{2}$ spaces has the best performance, which suggests that both $V_{1}$ and $V_{2}$ spaces are important for more complicated source functions.

\subsection{Adaptive enrichment algorithm.}

5.1.1. Adaptive enrichment algorithm with basis removal. In our adaptive enrichment algorithm, we can add basis functions to the offline space by using the error indicators. However, the addition of the basis functions must follow the ordering of the eigenfunctions. There may be cases that some of the intermediate eigenfunctions are not required in the representation of the solution. Therefore, we propose a numerical strategy to remove basis functions that do not contribute or contribute less to the representation of the solution. In the following, we will present this numerical strategy. Basically, the first four steps are the same as before. We introduce Step 5 below to remove unnecessary basis functions.

Copyright $\odot$ by SIAM. Unauthorized reproduction of this article is prohibited. 
Adaptive enrichment algorithm with basis Removal. Choose $0<\theta<1$. For each $m=0,1, \ldots$,

1. Step 1: Find the solution in the current space. That is, find $u_{H}^{m} \in V^{\text {off }}(m)$ such that

$$
a_{\mathrm{DG}}\left(u_{H}^{m}, v\right)=(f, v) \quad \forall v \in V^{\mathrm{off}}(m) .
$$

2. Step 2: Compute the local residuals. For each coarse grid block $K_{i}$, we compute

$$
\eta_{j, i}^{2}=\left\|R_{j, i}\right\|^{2}\left(\lambda_{j, l_{j, i}^{m}+1}^{(i)}\right)^{-1}, \quad j=1,2 .
$$

Then we reenumerate the $2 N$ residuals in the decreasing order, that is, $\eta_{1}^{2} \geq$ $\eta_{2}^{2} \geq \cdots \geq \eta_{2 N}^{2}$, where we adopted single index notation.

3. Step 3: Find the coarse grid blocks where enrichment is needed. We choose the smallest integer $k$ such that

$$
\theta \sum_{J=1}^{2 N} \eta_{J}^{2} \leq \sum_{J=1}^{k} \eta_{J}^{2}
$$

4. Step 4: Enrich the space. For each $J=1,2, \ldots, k$, we add a basis function in $V_{j}^{i, \text { off }}$ according to the following rule. Let $s$ be the smallest positive integer such that $\lambda_{j, l_{j, i}^{m}+s+1}^{(i)}$ is large enough (see the proof of Theorem 4.2) compared with $\lambda_{j, l_{j, i}^{m}+1}^{(i)}$. Then we include the eigenfunctions in the construction of the basis functions. The resulting space is denoted as $\widehat{V}^{\text {off }}(m+1)$. Note that this is the offline space without basis removal.

5. Step 5: Remove basis. In this step, we will remove basis functions that have little contribution to the solution. For each coarse grid block $K_{i}$, we can write the restriction of the current solution $u_{H}^{m}$ on $K_{i}$ as

$$
\sum_{l=1}^{l_{1, i}^{m}} \alpha_{1, l} \phi_{l}^{(i)}+\sum_{l=1}^{l_{2, i}^{m}} \alpha_{2, l} \xi_{l}^{(i)}
$$

Fixed a tolerance $\varepsilon>0$, which specify the importance of basis functions. Then the basis function $\phi_{l}^{(i)}$ or $\xi_{l}^{(i)}$ is removed from $\widehat{V}^{\text {off }}(m+1)$ if

$$
\alpha_{1, l}^{2}<\varepsilon\left(\sum_{l=1}^{l_{1, i}^{m}} \alpha_{1, l}^{2}+\sum_{l=1}^{l_{2, i}^{m}} \alpha_{2, l}^{2}\right) \quad \text { or } \quad \alpha_{2, l}^{2}<\varepsilon\left(\sum_{l=1}^{l_{1, i}^{m}} \alpha_{1, l}^{2}+\sum_{l=1}^{l_{2, i}^{m}} \alpha_{2, l}^{2}\right)
$$

is satisfied. The resulting space is called $V^{\text {off }}(m+1)$.

To test this strategy, we consider our second example with the source function $f$ defined in Figure 5. We will consider three choices of $\varepsilon$, with values $10^{-12}, 10^{-13}$, and $10^{-14}$. The convergence history of these cases are shown in Table 6 . We can see that our basis removal strategy gives more efficient representation of the solution. For example, comparing the errors with DOF of around 2000 with basis removal (Table 6 ) and without basis removal (Table 4), we see that the method with basis removal gives a solution with smaller errors in both $L^{2}$ norm and $a$-norm. On the other hand, we see that the choice of $\varepsilon=10^{-14}$ performs better than $\varepsilon=10^{-12}$. In particular, for DOF of around 2200, the error with $\varepsilon=10^{-14}$ is around $2 \%$, while the error with $\varepsilon=10^{-12}$ is around $4 \%$. However, one expects that smaller choices of $\varepsilon$ are not as economical as larger choices of $\varepsilon$. 
TABLE 6

Enrichment with $\theta=0.4$ and basis removal as well as enrichment in $V_{1}$ space only. Left: $\varepsilon=10^{-12}$. Middle: $\varepsilon=10^{-13}$. Right: $\varepsilon=10^{-14}$.

\begin{tabular}{|c|c|c|}
\hline DOF & $L_{2}$-error & $a$-error \\
\hline 1024 & 0.2052 & 0.0554 \\
\hline 951 & 0.1824 & 0.0502 \\
\hline 1074 & 0.1158 & 0.0415 \\
\hline 1742 & 0.0461 & 0.0174 \\
\hline 2218 & 0.0404 & 0.0153 \\
\hline
\end{tabular}

\begin{tabular}{|c|c|c|}
\hline DOF & $L_{2}$-error & $a$-error \\
\hline 1024 & 0.2052 & 0.0554 \\
\hline 996 & 0.1767 & 0.0501 \\
\hline 1107 & 0.1236 & 0.0431 \\
\hline 2006 & 0.0266 & 0.0154 \\
\hline 2824 & 0.0192 & 0.0123 \\
\hline
\end{tabular}

\begin{tabular}{|c|c|c|}
\hline DOF & $L_{2}$-error & $a$-error \\
\hline 1024 & 0.2052 & 0.0554 \\
\hline 1048 & 0.1774 & 0.0500 \\
\hline 1185 & 0.1280 & 0.0434 \\
\hline 2235 & 0.0223 & 0.0150 \\
\hline 3275 & 0.0147 & 0.0117 \\
\hline
\end{tabular}

5.1.2. Adaptive enrichment using local basis pursuit. In this section, we discuss an algorithm that follows basis pursuit ideas [4] and identify the basis functions which need to be added based on the residual. The main idea is to find multiscale basis functions that correlate to the residual the most and add those basis functions. More precisely, we identify basis functions that have the largest correlation coefficient with respect to the residual and add those basis functions. In the following, we will present the details of the numerical algorithm. The key components of the algorithm are Step 2 and Step 3, which determine the basis functions that correlate to the residual the most.

Adaptive enrichment Algorithm Using LOCAl Basis Pursuit. Choose $0<$ $\theta<1$. For each $m=0,1, \ldots$,

1. Step 1: Find the solution in the current space. That is, find $u_{H}^{m} \in V^{\text {off }}(m)$ such that

$$
a_{\mathrm{DG}}\left(u_{H}^{m}, v\right)=(f, v) \quad \forall v \in V^{\mathrm{off}}(m) .
$$

2. Step 2: Compute the local residuals. For each coarse grid block $K_{i}$, we compute

$$
\zeta_{j, i, l}^{2}=\frac{\left|R_{j, i}\left(v_{l}\right)\right|^{2}}{\left\|v_{l}\right\|_{V_{j}\left(K_{i}\right)}^{2}}, \quad j=1,2, \quad \forall v_{l} \in V_{j}\left(K_{i}\right) .
$$

Note that $\left|R_{j, i}\left(v_{l}\right)\right|$ is the inner product that identifies the basis functions that have the largest correlation to the residual. More precisely,

$$
\left|R_{j, i}\left(v_{l}\right)\right|=\left|\int_{K_{i}} f v_{l}-a_{D G}\left(u_{H}^{m}, v_{l}\right)\right|,
$$

which is just the local inner product of the residual vector and basis function $v_{l}$. We remark that $v_{l}$ denotes generically the $l$ th basis functions in the space $V_{j}\left(K_{i}\right)$. The above quantity $\zeta_{j, i, l}^{2}$ has triple indices. But we reenumerate these residuals using single indices in the decreasing order, that is, $\zeta_{1}^{2} \geq \zeta_{2}^{2} \geq \cdots$. More precisely, each single index residual $\eta_{k}^{2}$ corresponds to a triple index residual $\zeta_{j, i, l}^{2}$ for some $(j, i, l)$.

3. Step 3: Find the coarse grid blocks where enrichment is needed. We choose the smallest integer $k$ such that

$$
\zeta_{k} \geq \theta \zeta_{1}
$$

More precisely, if $\zeta_{k}^{2}$ is selected by (41), then by Step $2, \eta_{k}^{2}$ corresponds to a triple index residual $\zeta_{j, i, l}^{2}$ for some $(j, i, l)$. We will then add the basis function $v_{l}$ in the space $V_{j}\left(K_{i}\right)$ to the new approximation space. (See Step 4.) 


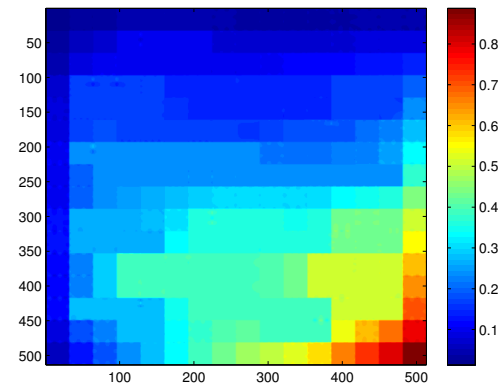

FIG. 7. Solution with sparse coefficient.

TABLE 7

Enrichment using basis pursuit with $\theta=0.8$ and basis removal as well as enrichment in $V_{1}$ space only.

\begin{tabular}{|c|c|c|}
\hline DOF & $e_{2}$ & $e_{a}$ \\
\hline 1024 & 0.2052 & 0.0554 \\
\hline 1036 & 0.1474 & 0.0405 \\
\hline 1259 & 0.0585 & 0.0230 \\
\hline 2096 & 0.0129 & 0.0125 \\
\hline 2643 & 0.0099 & 0.0111 \\
\hline
\end{tabular}

4. Step 4: Enrich the space. For each $J=1,2, \ldots, k$, we add the basis function $v_{l} \in V_{j}\left(K_{i}\right)$ corresponding to $\zeta_{J}$. The resulting space is denoted as $\widehat{V}^{\text {off }}(m+$ $1)$. Note that this is the offline space without basis removal.

5. Step 5: Remove basis. For each coarse grid block $K_{i}$, we can write the restriction of the current solution $u_{H}^{m}$ on $K_{i}$ as

$$
\sum_{l=1}^{l_{1, i}^{m}} \alpha_{1, l} \phi_{l}^{(i)}+\sum_{l=1}^{l_{2, i}^{m}} \alpha_{2, l} \xi_{l}^{(i)}
$$

Fixed a tolerance $\varepsilon>0$. Then the basis function $\phi_{l}^{(i)}$ or $\xi_{l}^{(i)}$ is removed if

$$
\alpha_{1, l}^{2}<\varepsilon\left(\sum_{l=1}^{l_{1, i}^{m}} \alpha_{1, l}^{2}+\sum_{l=1}^{l_{2, i}^{m}} \alpha_{2, l}^{2}\right) \quad \text { or } \quad \alpha_{2, l}^{2}<\varepsilon\left(\sum_{l=1}^{l_{1, i}^{m}} \alpha_{1, l}^{2}+\sum_{l=1}^{l_{2, i}^{m}} \alpha_{2, l}^{2}\right)
$$

is satisfied. The resulting space is called $V^{\mathrm{off}}(m+1)$.

To demonstrate the performance of this strategy, we will consider two examples. In the first example, the source function $f$ is defined as in Figure 5 and the rest of the parameters as in Example 2. In the second example, we will take the solution (see Figure 7) which only contains the component of the 1st, 17th, and 30th eigenbases. The boundary conditions are as in Example 2 and the source term is calculated based on this sparse solution. The convergence history for the first example is shown in Table 7. Comparing these results to Table 6 , we can see that the adaptive enrichment provides a better convergence. The convergence history is substantially improved if we consider the sparse solution as in our second example. The numerical results are shown in Table 8. 
TABLE 8

Enrichment with $\theta=0.8$. Left: basis pursuit. Right: standard enrichment.

\begin{tabular}{|c|c|c||c|c|c|}
\hline DOF & $e_{2}$ & $e_{a}$ & DOF & $e_{2}$ & $e_{a}$ \\
\hline 1024 & 0.0150 & 0.0424 \\
\hline 941 & 0.0069 & 0.0286 \\
\hline 934 & 0.0032 & 0.0135 \\
\hline 688 & 0.0001 & 0.0010 \\
\hline 744 & $1.20 \mathrm{e}-06$ & $2.13 \mathrm{e}-05$ \\
\hline 997 & 0.0150 & 0.0424 \\
\hline 1327 & 0.0150 & 0.0424 \\
\hline 2447 & 0.0023 & 0.0154 \\
\hline 889 & 0.0003 & 0.0022 \\
\hline
\end{tabular}

Remark 5.1. We remark that the method using local basis pursuit will work well when the solution contains only a few modes, that is, the solution is sparse. Under this assumption, the method will efficiently identify a few basis functions without adding eigenfunctions that are not needed. In terms of computational efficiency, both approaches are similar.

6. Convergence analysis. In this section, we will provide the proofs for the a posteriori error estimates (Theorem 4.1) and the convergence of the adaptive enrichment algorithm (Theorem 4.2).

For each coarse grid block $K_{i}, i=1,2, \ldots, N$, we define two projection operators $P_{j, i}: V_{j}^{i \text {,snap }} \rightarrow V_{j}^{i, \text { off }}, j=1,2$, from the local snapshot spaces to the corresponding local offline spaces by

$$
\begin{aligned}
\int_{\partial K_{i}} \widetilde{\kappa} P_{1, i}(v) w & =\int_{\partial K_{i}} \widetilde{\kappa} v w & \forall w \in V_{1}^{i, \mathrm{off}}, \\
\int_{K_{i}} \kappa P_{2, i}(v) w & =\int_{K_{i}} \kappa v w & \forall w \in V_{2}^{i, \mathrm{off}} .
\end{aligned}
$$

For any $v \in V_{1}^{i, \text { snap }}$, we can write $v=\sum_{l=1}^{M_{i}} c_{1, l} \phi_{l}^{(i)}$. By orthogonality of eigenfunctions, we have $P_{1, i}(v)=\sum_{l=1}^{l_{1, i}} c_{1, l} \phi_{l}^{(i)}$. Therefore, by the equivalence of $\|\cdot\|_{a}$ and $\|\cdot\|_{\mathrm{DG}}$, we have

$$
\left\|P_{1, i}(v)\right\|_{a}^{2} \leq a_{1}\left(\int_{K_{i}} \kappa\left|\nabla P_{1, i}(v)\right|^{2}+\frac{\gamma}{h} \int_{\partial K_{i}} \widetilde{\kappa} P_{1, i}(v)^{2}\right) .
$$

By the spectral problem (6) and the fact that the eigenvalues are ordered increasingly, we have

$$
\begin{aligned}
\left\|P_{1, i}(v)\right\|_{a}^{2} & \leq a_{1}\left(\sum_{l=1}^{l_{1, i}} \frac{\lambda_{1, l}^{(i)}}{H} c_{1, l}^{2}+\frac{\gamma}{h} \int_{\partial K_{i}} \widetilde{\kappa} P_{1, i}(v)^{2}\right) \\
& \leq a_{1}\left(\frac{\lambda_{1, l_{1, i}}^{(i)}}{H}+\frac{\gamma}{h}\right) \sum_{l=1}^{l_{1, i}} c_{1, l}^{2}=a_{1}\left(\lambda_{1, l_{1, i}}^{(i)}+\frac{\gamma H}{h}\right)\|v\|_{V_{1}\left(K_{i}\right)}^{2} .
\end{aligned}
$$

Similarly, for $v=\sum_{l \geq 1} c_{2, l} \xi_{l}^{(i)}$, we have $P_{2, i}(v)=\sum_{l=1}^{l_{2, i}} c_{2, l} \xi_{l}^{(i)}$. Therefore, by the equivalence of $\|\cdot\|_{a}$ and $\|\cdot\|_{\mathrm{DG}}$, we have

$$
\left\|P_{2, i}(v)\right\|_{a}^{2} \leq a_{1}\left(\int_{K_{i}} \kappa\left|\nabla P_{2, i}(v)\right|^{2}\right) .
$$

By the spectral problem (7) and the fact that the eigenvalues are ordered increasingly,

Copyright $\odot$ by SIAM. Unauthorized reproduction of this article is prohibited. 
we have

$$
\left\|P_{2, i}(v)\right\|_{a}^{2} \leq a_{1}\left(\sum_{l=1}^{l_{2, i}} \frac{\lambda_{2, l}^{(i)}}{H^{2}} c_{2, l}^{2}\right) \leq a_{1}\left(\frac{\lambda_{2, l_{2, i}}^{(i)}}{H^{2}}\right) \sum_{l=1}^{l_{2, i}} c_{2, l}^{2}=a_{1} \lambda_{2, l_{2, i}}^{(i)}\|v\|_{V_{2}\left(K_{i}\right)}^{2} .
$$

Thus the projections $P_{j, i}$ satisfy the following stability bound:

$$
\left\|P_{j, i}(v)\right\|_{a} \leq a_{1}^{\frac{1}{2}}\left(\lambda_{j, l_{j, i}}^{(i)}+\frac{\gamma H}{h}\right)^{\frac{1}{2}}\|v\|_{V_{j}\left(K_{i}\right)}, \quad j=1,2, \quad i=1,2, \ldots, N .
$$

Next, we will establish some approximation properties for the projection operators $P_{j, i}$. Indeed, by the definitions of the operators $P_{j, i}$, for any $v \in V_{j}^{i, \text { snap }}$,

$$
\begin{aligned}
\left\|v-P_{j, i}(v)\right\|_{V_{j}\left(K_{i}\right)}^{2} & =H^{-j} \sum_{l \geq l_{j, i}+1} c_{j, l}^{2} \leq\left(\lambda_{l_{j, i}+1}^{(i)}\right)^{-1} \sum_{l \geq l_{j, i}+1} \frac{\lambda_{j, l}^{(i)}}{H^{j}} c_{j, l}^{2} \\
& =\left(\lambda_{j, l_{j, i}+1}^{(i)}\right)^{-1} \int_{K_{i}} \kappa|\nabla v|^{2},
\end{aligned}
$$

and therefore the following convergence result holds:

$$
\left\|v-P_{j, i}(v)\right\|_{V_{j}\left(K_{i}\right)} \leq\left(\lambda_{j, l_{j, i}+1}^{(i)}\right)^{-\frac{1}{2}}\left(\int_{K_{i}} \kappa|\nabla v|^{2}\right)^{\frac{1}{2}} .
$$

For the analysis presented below, we define the projection $\Pi: V^{\text {snap }} \rightarrow V^{\text {off }}$ by $\Pi v=\sum_{i=1}^{N} \sum_{j=1}^{2} P_{j, i}(v)$.

6.1. Proof of Theorem 4.1. Let $v \in V_{\mathrm{DG}}^{h}$ be an arbitrary function in the space $V_{\mathrm{DG}}^{h}$. Using (27), we have

$$
a_{\mathrm{DG}}\left(u_{h}-u_{H}, v\right)=a_{\mathrm{DG}}\left(u_{h}, v\right)-a_{\mathrm{DG}}\left(u_{H}, v\right)=(f, v)-a_{\mathrm{DG}}\left(u_{H}, v\right) .
$$

Therefore,

$$
\begin{aligned}
a_{\mathrm{DG}}\left(u_{h}-u_{H}, v\right) & =(f, v)-a_{\mathrm{DG}}\left(u_{H}, v\right) \\
& =(f, v-\Pi v)+(f, \Pi v)-a_{\mathrm{DG}}\left(u_{H}, \Pi v\right)-a_{\mathrm{DG}}\left(u_{H}, v-\Pi v\right) .
\end{aligned}
$$

Thus, using (28), we have

$$
a_{\mathrm{DG}}\left(u_{h}-u_{H}, v\right)=(f, v-\Pi v)-a_{\mathrm{DG}}\left(u_{H}, v-\Pi v\right) .
$$

Since the space $V_{\mathrm{DG}}^{h}$ is the same as $V^{\text {snap }}$, we can write $v=\sum_{i=1}^{N} \sum_{j=1}^{2} v_{j}^{(i)}$ with $v_{j}^{(i)} \in V_{j}^{i, \text { snap }}$. Hence, (44) becomes

$$
a_{\mathrm{DG}}\left(u_{h}-u_{H}, v\right)=\sum_{i=1}^{N} \sum_{j=1}^{2}\left(\int_{K_{i}} f\left(v_{j}^{(i)}-P_{j, i} v_{j}^{(i)}\right)-a_{\mathrm{DG}}\left(u_{H}, v_{j}^{(i)}-P_{j, i} v_{j}^{(i)}\right)\right) .
$$

We remark that, in the computation of the term $a_{\mathrm{DG}}\left(u_{H}, v_{j}^{(i)}-P_{j, i} v_{j}^{(i)}\right)$ in (45), we assume that the second argument is zero outside the coarse grid block $K_{i}$.

Copyright $@$ ㅇ by SIAM. Unauthorized reproduction of this article is prohibited. 
Using the definition of $R_{j, i}$, we see that (45) can be written as

$$
a_{\mathrm{DG}}\left(u_{h}-u_{H}, v\right)=\sum_{i=1}^{N} \sum_{j=1}^{2} R_{j, i}\left(v_{j}^{(i)}-P_{j, i} v_{j}^{(i)}\right) .
$$

Thus, we have

$$
a_{\mathrm{DG}}\left(u_{h}-u_{H}, v\right) \leq \sum_{i=1}^{N} \sum_{j=1}^{2}\left\|R_{j, i}\right\|\left\|\left(v_{j}^{(i)}-P_{j, i} v_{j}^{(i)}\right)\right\|_{V_{j}\left(K_{i}\right)} .
$$

Using (43),

$$
a_{\mathrm{DG}}\left(u_{h}-u_{H}, v\right) \leq \sum_{i=1}^{N} \sum_{j=1}^{2}\left\|R_{j, i}\right\|\left(\lambda_{j, l_{j, i}+1}^{(i)}\right)^{-\frac{1}{2}}\left(\int_{K_{i}} \kappa\left|\nabla v_{j}^{(i)}\right|^{2}\right)^{\frac{1}{2}} .
$$

The inequality (33) is then followed by taking $v=u_{h}-u_{H}$ and $\sum_{i=1}^{N} \sum_{j=1}^{2} \int_{K_{i}} \kappa\left|\nabla v_{j}^{(i)}\right|^{2} \leq\|v\|_{\mathrm{DG}}^{2} \leq a_{0}\|v\|_{a}^{2}$.

6.2. An auxiliary lemma. In this section, we will derive an auxiliary lemma which will be used for the proof of the convergence of the adaptive enrichment algorithm stated in Theorem 4.2. We use the notation $P_{j, i}^{m}$ to denote the projection operator $P_{j, i}$ at the enrichment level $m$.

In Theorem 4.1, we see that $\left\|R_{j, i}\right\|$ gives an upper bound of the energy error $\left\|u_{h}-u_{H}\right\|_{a}$. We will first show that $\left\|R_{j, i}\right\|$ is also a lower bound up to a correction term (see Lemma 6.1). To state this precisely, we define

$$
S_{j, i}^{m}=\left(\lambda_{j, l_{j, i}^{m}+1}^{(i)}\right)^{-\frac{1}{2}} \sup _{v \in V_{j}^{i, \text { snap }}} \frac{\left|R_{j, i}\left(v-P_{j, i}^{m}(v)\right)\right|}{\|v\|_{V_{j}\left(K_{i}\right)}} .
$$

Notice that the residual $R_{j, i}$ is computed using the solution $u_{H}^{m}$ obtained at enrichment level $m$. We omit the index $m$ in $R_{j, i}$ to simplify notation. Next, we will obtain

$$
\left(S_{j, i}^{m}\right)^{2}=\left\|R_{j, i}\right\|^{2}\left(\lambda_{j, l_{j, i}^{m}+1}^{(i)}\right)^{-1} .
$$

Indeed, by the fact that $P_{j, i}^{m}(v) \in V_{j}^{i, \text { off }}$,

$$
R_{j, i}\left(P_{j, i}^{m}(v)\right)=\int_{K_{i}} f P_{j, i}^{m}(v)-a_{\mathrm{DG}}\left(u_{H}^{m}, P_{j, i}^{m}(v)\right)=0 .
$$

Thus,

$$
\begin{aligned}
S_{j, i}^{m}=\left(\lambda_{j, l_{j, i}^{m}+1}^{(i)}\right)^{-\frac{1}{2}} \sup _{v \in V_{j}^{i, \text { snap }}} \frac{\left|R_{j, i}\left(v-P_{j, i}^{m}(v)\right)\right|}{\|v\|_{V_{j}\left(K_{i}\right)}} & =\left(\lambda_{j, l_{j, i}^{m}+1}^{(i)}\right)^{-\frac{1}{2}} \sup _{v \in V_{j}^{i, \text { snap }}} \frac{\left|R_{j, i}(v)\right|}{\|v\|_{V_{j}\left(K_{i}\right)}} \\
& =\left(\lambda_{j, l_{j, i}^{m}+1}^{(i)}\right)^{-\frac{1}{2}}\left\|R_{j, i}\right\| .
\end{aligned}
$$

This implies (47).

To prove Theorem 4.2, we will need the following recursive properties for $S_{j, i}^{m}$ (see Lemma 6.1). Notice that the notation $\|u\|_{a, K_{i}}$ is defined as

$$
\|u\|_{a, K_{i}}^{2}=a_{\mathrm{DG}}(u, u)=\int_{K_{i}} \kappa|\nabla u|^{2}-\sum_{E \in \partial K_{i}} 2 \int_{E}\left\{\kappa \nabla u \cdot n_{E}\right\} \llbracket u \rrbracket+\sum_{E \in \partial K_{i}} \frac{\gamma}{h} \int_{E} \bar{\kappa} \llbracket u \rrbracket^{2} .
$$

Copyright $@$ by SIAM. Unauthorized reproduction of this article is prohibited. 
Lemma 6.1. For any $\alpha>0$, we have

$$
\left(S_{j, i}^{m+1}\right)^{2} \leq(1+\alpha) \frac{\lambda_{j, l_{j, i}^{m}+1}^{(i)}}{\lambda_{j, l_{j, i}^{m+1}+1}^{(i)}}\left(S_{j, i}^{m}\right)^{2}+\left(1+\alpha^{-1}\right) a_{1} D\left\|u_{H}^{m+1}-u_{H}^{m}\right\|_{a, K_{i}}^{2},
$$

where the enrichment level dependent constant $D$ is defined by

$$
D=\left(\frac{\Lambda_{j, i}}{\lambda_{j, l_{j, i}^{m+1}}^{(i)}}+\frac{\gamma H}{h \lambda_{j, l_{j, i}^{m+1}+1}^{(i)}}\right)
$$

with $\Lambda_{j, i}=\max _{l} \lambda_{j, l}^{(i)}$.

Proof. By direct calculations, we have

$$
\begin{aligned}
& \int_{K_{i}} f\left(v-P_{j, i}^{m+1}(v)\right)-a_{\mathrm{DG}}\left(u_{H}^{m+1}, v-P_{j, i}^{m+1}(v)\right) \\
= & \int_{K_{i}} f v-a_{\mathrm{DG}}\left(u_{H}^{m+1}, v\right) \\
= & \int_{K_{i}} f v-a_{\mathrm{DG}}\left(u_{H}^{m}, v\right)+a_{\mathrm{DG}}\left(u_{H}^{m}-u_{H}^{m+1}, v\right) \\
= & \int_{K_{i}} f\left(v-P_{j, i}^{m}(v)\right)-a_{\mathrm{DG}}\left(u_{H}^{m}, v-P_{j, i}^{m}(v)\right)+a_{\mathrm{DG}}\left(u_{H}^{m}-u_{H}^{m+1}, v\right) .
\end{aligned}
$$

By definition of $S_{j, i}^{m}$, we have

$$
S_{j, i}^{m}=\left(\lambda_{j, l_{j, i}^{m}+1}^{(i)}\right)^{-\frac{1}{2}} \sup _{v \in V_{j}^{i, \text { snap }}} \frac{\left|\int_{K_{i}} f\left(v-P_{j, i}^{m}(v)\right)-a_{\mathrm{DG}}\left(u_{H}^{m}, v-P_{j, i}^{m}(v)\right)\right|}{\|v\|_{V_{j}\left(K_{i}\right)}} .
$$

Multiplying (49) by $\left(\lambda_{j, l_{j, i}^{m}+1}^{(i)}\right)^{-\frac{1}{2}}\|v\|_{V_{j}\left(K_{i}\right)}^{-1}$ and taking supremum with respect to $v$, we have

$$
S_{j, i}^{m+1} \leq\left(\frac{\lambda_{j, l_{j, i}^{m}+1}^{(i)}}{\lambda_{j, l_{j, i}^{m+1}+1}^{(i)}}\right)^{\frac{1}{2}} S_{j, i}^{m}+I
$$

where

$$
I=\left(\lambda_{j, l_{j, i}^{m}+1}^{(i)}\right)^{-\frac{1}{2}} \sup _{v \in V_{j}^{i, \text { snap }}} \frac{\left|a_{\mathrm{DG}}\left(u_{H}^{m}-u_{H}^{m+1}, v\right)\right|}{\|v\|_{V_{j}\left(K_{i}\right)}} .
$$

To estimate $I$, we note that

$$
a_{\mathrm{DG}}\left(u_{H}^{m}, P_{j, i}^{m}(v)\right)=\int_{K_{i}} f P_{j, i}^{m}(v)=a_{\mathrm{DG}}\left(u_{H}^{m+1}, P_{j, i}^{m}(v)\right) .
$$

Therefore, we have

$a_{\mathrm{DG}}\left(u_{H}^{m}-u_{H}^{m+1}, v\right)=a_{\mathrm{DG}}\left(u_{H}^{m}-u_{H}^{m+1}, v-P_{j, i}^{m}(v)\right) \leq\left\|u_{H}^{m}-u_{H}^{m+1}\right\|_{a, K_{i}}\left\|v-P_{j, i}^{m}(v)\right\|_{a, K_{i}}$,

Copyright $@$ by SIAM. Unauthorized reproduction of this article is prohibited. 
where we remark that $v$ has value zero outside $K_{i}$. By the stability bound (42),

$$
\left\|v-P_{j, i}^{m}(v)\right\|_{a} \leq a_{1}^{\frac{1}{2}}\left(\Lambda_{j, i}+\frac{\gamma H}{h}\right)^{\frac{1}{2}}\left\|v-P_{j, i}^{m}(v)\right\|_{V_{j}\left(K_{i}\right)} \leq a_{1}^{\frac{1}{2}}\left(\Lambda_{j, i}+\frac{\gamma H}{h}\right)^{\frac{1}{2}}\|v\|_{V_{j}\left(K_{i}\right)} .
$$

Thus we have

$$
I \leq a_{1}^{\frac{1}{2}}\left(\lambda_{j, l_{j, i}^{m}+1}^{(i)}\right)^{-\frac{1}{2}}\left(\Lambda_{j, i}+\frac{\gamma H}{h}\right)^{\frac{1}{2}}\left\|u_{H}^{m+1}-u_{H}^{m}\right\|_{a, K_{i}} .
$$

Using (51), we get

$$
S_{j, i}^{m+1} \leq\left(\frac{\lambda_{j, l_{j, i}^{m}+1}^{(i)}}{\lambda_{j, l_{j, i}^{m}+1}^{(i)+1}}\right)^{\frac{1}{2}} S_{j, i}^{m}+a_{1}^{\frac{1}{2}}\left(\frac{\Lambda_{j, i}}{\lambda_{j, l_{j, i}^{(i)}+1}^{m+1}+1}+\frac{\gamma H}{h \lambda_{j, l_{j, i}^{m+1}+1}^{(i)}}\right)^{\frac{1}{2}}\left\|u_{\mathrm{ms}}^{m+1}-u_{\mathrm{ms}}^{m}\right\|_{a, K_{i}} .
$$

Hence, (48) is proved.

6.3. Proof of Theorem 4.2. In this section, we prove the convergence of the adaptive enrichment algorithm. First of all, we recall that

$$
\eta_{j, i}^{2}=\left\|R_{j, i}\right\|^{2}\left(\lambda_{j, l_{j, i}^{m}+1}^{(i)}\right)^{-1}=\left(S_{j, i}^{m}\right)^{2} .
$$

We let the single index $J=(j, i)$, and we will use the single index notation $\eta_{J}$ and $S_{J}^{m}$ for $\eta_{j, i}$ and $S_{j, i}^{m}$, respectively. Note that there is a one-to-one mapping of the set of single indices $J$ and the set of integers $\{1,2, \ldots, 2 N\}$.

Let $0<\theta<1$. We choose an index set $I$ so that

$$
\theta^{2} \sum_{J=1}^{2 N} \eta_{J}^{2} \leq \sum_{J \in I} \eta_{J}^{2}
$$

We will then add the basis function from $V_{j}^{i, \text { snap }}$ with $J=(j, i) \in I$. Then, using Theorem 4.1 and (53), we have

$$
\theta^{2}\left\|u_{h}-u_{H}^{m}\right\|_{a}^{2} \leq \theta^{2} C_{\text {err }} \sum_{J=1}^{2 N} \eta_{J}^{2} \leq C_{\text {err }} \sum_{J \in I} \eta_{J}^{2}
$$

By (52), we also have

$$
\left\|u_{h}-u_{H}^{m}\right\|_{a}^{2} \leq \frac{C_{\mathrm{err}}}{\theta^{2}} \sum_{J \in I}\left(S_{J}^{m}\right)^{2}
$$

On the other hand,

$$
\sum_{J=1}^{N}\left(S_{J}^{m+1}\right)^{2}=\sum_{J \in I}\left(S_{J}^{m+1}\right)^{2}+\sum_{J \notin I}\left(S_{J}^{m+1}\right)^{2} .
$$

By Lemma 6.1 , for any $J \in I$, we have

$$
\left(S_{J}^{m+1}\right)^{2} \leq(1+\alpha) \frac{\lambda_{j, l_{j, i}^{m}+1}^{(i)}}{\lambda_{j, l_{j, i}^{m}+1}^{(i)}}\left(S_{J}^{m}\right)^{2}+\left(1+\alpha^{-1}\right) a_{1} D\left\|u_{H}^{m+1}-u_{H}^{m}\right\|_{a, K_{i}}^{2} .
$$

Copyright (c) by SIAM. Unauthorized reproduction of this article is prohibited. 
If $J \notin I$, then there is no enrichment involved. So $\lambda_{j, l_{j, i}^{m}+1}^{(i)}=\lambda_{j, l_{j, i}^{m+1}+1}^{(i)}$, which implies

$$
\left(S_{J}^{m+1}\right)^{2} \leq(1+\alpha)\left(S_{J}^{m}\right)^{2}+\left(1+\alpha^{-1}\right) a_{1} D\left\|u_{H}^{m+1}-u_{H}^{m}\right\|_{a, K_{i}}^{2} .
$$

Combining the above two cases, we have

$$
\begin{aligned}
\sum_{J=1}^{2 N}\left(S_{J}^{m+1}\right)^{2} \leq & \sum_{J \in I}\left((1+\alpha) \frac{\lambda_{j, l_{j, i}^{m}+1}^{(i)}}{\lambda_{j, l_{j, i}^{m}+1}^{(i)}+1}\left(S_{J}^{m}\right)^{2}+\left(1+\alpha^{-1}\right) a_{1} D\left\|u_{H}^{m+1}-u_{H}^{m}\right\|_{a, K_{i}}^{2}\right) \\
& +\sum_{J \notin I}\left((1+\alpha)\left(S_{J}^{m}\right)^{2}+\left(1+\alpha^{-1}\right) a_{1} D\left\|u_{H}^{m+1}-u_{H}^{m}\right\|_{a, K_{i}}^{2}\right) .
\end{aligned}
$$

We assume the enrichment is obtained so that

$$
\delta:=\max _{J \in I} \frac{\lambda_{j, l_{j, i}^{m}+1}^{(i)}}{\lambda_{j, l_{j, i}^{m+1}+1}^{(i)}} \leq \delta_{0}<1
$$

where $\delta_{0}$ is independent of $m$. We then have

$$
\begin{aligned}
\sum_{J=1}^{2 N}\left(S_{J}^{m+1}\right)^{2} \leq & \sum_{J \in I}\left((1+\alpha) \delta_{0}\left(S_{J}^{m}\right)^{2}\right) \\
& +\sum_{J \notin I}\left((1+\alpha)\left(S_{J}^{m}\right)^{2}\right)+\sum_{J=1}^{2 N}\left(1+\alpha^{-1}\right) a_{1} D\left\|u_{H}^{m+1}-u_{H}^{m}\right\|_{a, K_{i}}^{2} .
\end{aligned}
$$

Since $\delta_{0}=1-\left(1-\delta_{0}\right)$, the above can be written as

$$
\sum_{J=1}^{2 N}\left(S_{J}^{m+1}\right)^{2} \leq(1+\alpha) \sum_{J=1}^{2 N}\left(S_{J}^{m}\right)^{2}-(1+\alpha)\left(1-\delta_{0}\right) \sum_{J \in I}\left(S_{J}^{m}\right)^{2}+L_{m+1}\left\|u_{H}^{m+1}-u_{H}^{m}\right\|_{a}^{2},
$$

where

$$
L_{m+1}=N_{E}\left(1+\alpha^{-1}\right) a_{1}\left(\max _{1 \leq i \leq N} \max _{1 \leq j \leq 2} D\right),
$$

where $N_{E}$ is the maximum number of edges of coarse grid blocks, and we also emphasize that $L_{m+1}$ depends on $m$. By (53),

$$
\sum_{J=1}^{2 N}\left(S_{J}^{m+1}\right)^{2} \leq(1+\alpha) \sum_{J=1}^{2 N}\left(S_{J}^{m}\right)^{2}-(1+\alpha)\left(1-\delta_{0}\right) \theta^{2} \sum_{J=1}^{2 N}\left(S_{J}^{m}\right)^{2}+L_{m+1}\left\|u_{H}^{m+1}-u_{H}^{m}\right\|_{a}^{2} .
$$

Let $\rho=(1+\alpha)\left(1-\left(1-\delta_{0}\right) \theta^{2}\right)$. We choose $\alpha>0$ small enough so that $0<\rho<1$. We remark that $\alpha$ is fixed if we fix the values of $\delta_{0}$ and $\theta$, which are user inputs. The above inequality can then be written as

$$
\sum_{J=1}^{2 N}\left(S_{J}^{m+1}\right)^{2} \leq \rho \sum_{J=1}^{2 N}\left(S_{J}^{m}\right)^{2}+L_{m+1}\left\|u_{H}^{m+1}-u_{H}^{m}\right\|_{a}^{2}
$$

Note that, by Galerkin orthogonality, we have

$$
\left\|u_{H}^{m+1}-u_{H}^{m}\right\|_{a}^{2}=\left\|u_{h}-u_{H}^{m}\right\|_{a}^{2}-\left\|u_{h}-u_{H}^{m+1}\right\|_{a}^{2}
$$

Copyright (c) by SIAM. Unauthorized reproduction of this article is prohibited. 
We let $\tau>0$, which will be specified in the following. Then, by using (51) and (57), we have

$$
\left\|u_{h}-u_{H}^{m+1}\right\|_{a}^{2}+\tau \sum_{J=1}^{2 N}\left(S_{J}^{m+1}\right)^{2} \leq\left\|u_{h}-u_{H}^{m}\right\|_{a}^{2}+\tau \rho \sum_{J=1}^{2 N}\left(S_{J}^{m}\right)^{2}+\tau L_{m+1}\left\|u_{H}^{m+1}-u_{H}^{m}\right\|_{a}^{2} .
$$

Using (57), the above can be written as

$$
\begin{aligned}
& \left\|u_{h}-u_{H}^{m+1}\right\|_{a}^{2}+\tau \sum_{J=1}^{2 N}\left(S_{J}^{m+1}\right)^{2} \\
& \leq\left\|u_{h}-u_{H}^{m}\right\|_{a}^{2}+\tau \rho \sum_{J=1}^{2 N}\left(S_{J}^{m}\right)^{2}+\tau L_{m+1}\left(\left\|u_{h}-u_{H}^{m}\right\|_{a}^{2}-\left\|u_{h}-u_{H}^{m+1}\right\|_{a}^{2}\right),
\end{aligned}
$$

which implies

$$
\left\|u_{h}-u_{H}^{m+1}\right\|_{a}^{2}+\frac{\tau}{1+\tau L_{m+1}} \sum_{J=1}^{2 N}\left(S_{J}^{m+1}\right)^{2} \leq\left\|u_{h}-u_{H}^{m}\right\|_{a}^{2}+\frac{\tau \rho}{1+\tau L_{m+1}} \sum_{J=1}^{2 N}\left(S_{J}^{m}\right)^{2} .
$$

Next, we let $0<\beta<1$, which will be specified in the following. Using (54), we have

$$
\begin{aligned}
& \left\|u_{h}-u_{H}^{m+1}\right\|_{a}^{2}+\frac{\tau}{1+\tau L_{m+1}} \sum_{J=1}^{2 N}\left(S_{J}^{m+1}\right)^{2} \\
& \quad \leq(1-\beta)\left\|u_{h}-u_{H}^{m}\right\|_{a}^{2}+\left(\frac{\beta C_{\mathrm{err}}}{\theta^{2}}+\frac{\tau \rho}{1+\tau L_{m+1}}\right) \sum_{J=1}^{2 N}\left(S_{J}^{m}\right)^{2} .
\end{aligned}
$$

We choose $\beta$ by the following:

$$
\beta=\theta^{2}\left(\theta^{2}+C_{\mathrm{err}} \tau^{-1}\left(1+\tau L_{m}\right)\right)^{-1}\left(1-\rho \frac{1+\tau L_{m}}{1+\tau L_{m+1}}\right) .
$$

Then (58) becomes

$$
\left\|u_{h}-u_{H}^{m+1}\right\|_{a}^{2}+\frac{\tau}{1+\tau L_{m+1}} \sum_{J=1}^{2 N}\left(S_{J}^{m+1}\right)^{2} \leq(1-\beta)\left\|u_{h}-u_{H}^{m}\right\|_{a}^{2}+\frac{\tau(1-\beta)}{1+\tau L_{m}} \sum_{J=1}^{2 N}\left(S_{J}^{m}\right)^{2} .
$$

Finally, we will see that one can choose $\tau$ such that $\beta>0$. Since $\left\{L_{m}\right\}$ is a decreasing sequence, we have

$$
\frac{1+\tau L_{m}}{1+\tau L_{m+1}} \leq 1+\tau L_{1}
$$

Then we have

$$
1-\rho \frac{1+\tau L_{m}}{1+\tau L_{m+1}} \geq \epsilon_{0}>0 \quad \Longleftrightarrow \quad \tau<\frac{1-\rho-\epsilon_{0}}{L_{1}},
$$

where we take $\epsilon_{0}<1-\rho$. Using the above choice of $\tau$, we see that $\beta>0$. On the other hand, the condition $\beta<1$ is obvious. To complete the proof, we let $M$ 
be the maximum number of adaptive iterations, so that we have $L_{M}=a_{1} N_{E}(1+$ $\left.\alpha^{-1}\right) \max _{1 \leq i \leq N} \max _{1 \leq j \leq 2}\left(1+\gamma H h^{-1} \Lambda_{j, i}^{-1}\right)$. Using the condition (36), we obtain

$\beta \geq \epsilon_{0} \theta^{2}\left(C_{\mathrm{err}} \tau^{-1}\left(1+\tau L_{M}\right)+C_{\mathrm{err}} \tau^{-1}\left(1+\tau L_{m}\right)\right)^{-1} \geq \epsilon_{0} \theta^{2}\left(2 C_{\mathrm{err}} \tau^{-1}\left(1+\tau L_{M}\right)\right)^{-1}$,

which gives the required convergence rate $\epsilon$. This completes the proof of Theorem 4.2 .

\section{REFERENCES}

[1] A. Abdulle AND Y. BAI, Adaptive reduced basis finite element heterogeneous multiscale method, Comput. Methods Appl. Mech. Engrg., 257 (2013), pp. 203-220, https://doi.org/ 10.1016/j.cma.2013.01.002.

[2] T. Arbogast, Analysis of a two-scale, locally conservative subgrid upscaling for elliptic problems, SIAM J. Numer. Anal., 42 (2004), pp. 576-598, https://doi.org/10.1137/ S0036142902406636.

[3] S. Brenner and L. Scott, The Mathematical Theory of Finite Element Methods, Springer, New York, 2007.

[4] S. S. Chen, D. L. Donoho, and M. A. Saunders, Atomic decomposition by basis pursuit, SIAM Rev., 43 (2001), pp. 129-159, https://doi.org/10.1137/S003614450037906X.

[5] C.-C. Chu, I. G. Graham, and T.-Y. Hou, A new multiscale finite element method for highcontrast elliptic interface problems, Math. Comp., 79 (2010), pp. 1915-1955, https://doi. org/10.1090/S0025-5718-2010-02372-5.

[6] E. Chung And Y. Efendiev, Reduced-contrast approximations for high-contrast multiscale flow problems, Multiscale Model. Simul., 8 (2010), pp. 1128-1153.

[7] E. Chung, Y. Efendiev, And R. Gibson, An energy-conserving discontinuous multiscale finite element method for the wave equation in heterogeneous media, Adv. Adapt. Data Anal., 3 (2011), pp. 251-268.

[8] E. Chung, Y. Efendiev, And T. Y. Hou, Adaptive multiscale model reduction with generalized multiscale finite element methods, J. Comput. Phys., 320 (2016), pp. 69-95.

[9] E. Chung, Y. Efendiev, And W. T. Leung, Generalized Multiscale Finite Element Method for Wave Propagation in Heterogeneous Media, arXiv:1307.0123, 2013.

[10] E. Chung, Y. Efendiev, And G. Li, An adaptive GMsFEM for high contrast flow problems, J. Comput. Phys., 273 (2014), pp. 54-76.

[11] E. Chung AND W. T. LeUnG, A sub-grid structure enhanced discontinuous galerkin method for multiscale diffusion and convection-diffusion problems, Commun. Comput. Phys., 14 (2013), pp. 370-392.

[12] W. DoRfLER, A convergent adaptive algorithm for poisson's equation, SIAM J. Numer. Anal., 33 (1996), pp. 1106-1124.

[13] M. Drohmann, B. HaAsdonk, And M. OhlBerger, Reduced basis approximation for nonlinear parametrized evolution equations based on empirical operator interpolation, SIAM J. Sci. Comput., 34 (2012), pp. A937-A969, https://doi.org/10.1137/10081157X.

[14] L. DuRlofsky, Numerical calculation of equivalent grid block permeability tensors for heterogeneous porous media, Water Resour. Res., 27 (1991), pp. 699-708.

[15] W. E And B. Engquist, Heterogeneous multiscale methods, Commun. Math. Sci., 1 (2003), pp. $87-132$.

[16] Y. Efendiev, J. Galvis, and T. Hou, Generalized multiscale finite element methods, J. Comput. Phys., 251 (2013), pp. 116-135.

[17] Y. Efendiev, J. Galvis, R. Lazarov, M. Moon, and M. Sarkis, Generalized multiscale finite element method. Symmetric interior penalty coupling, J. Comput. Phys., 255 (2013), pp. 1-15, https://doi.org/10.1016/j.jcp.2013.07.028.

[18] Y. Efendiev, J. Galvis, G. Li, And M. Presho, Generalized multiscale finite element methods. oversampling strategies, Internat. J. Multiscale Comput. Engrg., 12 (2014), pp. 465-484.

[19] Y. Efendiev, J. Galvis, And X. Wu, Multiscale finite element methods for high-contrast problems using local spectral basis functions, J. Comput. Phys., 230 (2011), pp. 937-955.

[20] Y. Efendiev and T. Hou, Multiscale Finite Element Methods: Theory and Applications, Surv. Tutor. Appl. Math. Sci. 4, Springer, New York, 2009.

[21] Y. Efendiev, T. Hou, And V. Ginting, Multiscale finite element methods for nonlinear problems and their applications, Commun. Math. Sci., 2 (2004), pp. 553-589.

Copyright $@$ by SIAM. Unauthorized reproduction of this article is prohibited. 
[22] M. Ghommem, M. Presho, V. M. Calo, and Y. Efendiev, Mode decomposition methods for flows in high-contrast porous media. Global-local approach, J. Comput. Phys., 253 (2013), pp. 226-238.

[23] T. Hou And X. Wu, A multiscale finite element method for elliptic problems in composite materials and porous media, J. Comput. Phys., 134 (1997), pp. 169-189.

[24] D. B. P. Huynh, D. J. Knezevic, And A. T. PAtera, A static condensation reduced basis element method: approximation and a posteriori error estimation, ESAIM Math. Model. Numer. Anal., 47 (2013), pp. 213-251, https://doi.org/10.1051/m2an/2012022.

[25] K. Meксhay and R. H. Nochetto, Convergence of adaptive finite element method for general second order elliptic PDEs, SIAM J. Numer. Anal., 43 (2005), pp. 1803-1827.

[26] N. C. Nguyen, G. Rozza, D. B. P. Huynh, and A. T. Patera, Reduced basis approximation and a posteriori error estimation for parametrized parabolic PDEs: Application to realtime Bayesian parameter estimation, in Large-Scale Inverse Problems and Quantification of Uncertainty, Wiley Ser. Comput. Stat., Wiley, Chichester, UK, 2011, pp. 151-177.

[27] B. M. Riviere, Discontinuous Galerkin Methods for Solving Elliptic and parabolic Equations: Theory and Implementation, Front. Appl. Math. 85, SIAM, Philadelphia, 2008.

[28] T. Tonn, K. Urban, AND S. Volkwein, Comparison of the reduced-basis and POD a posteriori error estimators for an elliptic linear-quadratic optimal control problem, Math. Comput. Model. Dyn. Syst., 17 (2011), pp. 355-369, https://doi.org/10.1080/13873954.2011.547678.

[29] X. Wu, Y. Efendiev, AND T. Hou, Analysis of upscaling absolute permeability, Discrete Contin. Dyn. Syst. Ser. B, 2 (2002), pp. 158-204.

Copyright (c) by SIAM. Unauthorized reproduction of this article is prohibited. 